\title{
Complex Quantum Groups and Their Real Representations
}

By

\author{
Piotr PODLEś *
}

\begin{abstract}
We define *-Hopf algebras $F u n\left(S L_{q}\left(N, \mathbb{C} ; \varepsilon_{1}, \ldots, \varepsilon_{N}\right)\right), F u n\left(O_{q}\left(N, \mathbb{C} ; \varepsilon_{1}, \ldots, \varepsilon_{N}\right)\right)$ and $F u n\left(S p_{q}(n, \mathbb{C}\right.$; $\left.\left.\varepsilon_{1}, \ldots, \varepsilon_{2 n}\right)\right)$ as the real complexifications of $*$-Hopf algebras $F u n\left(S U_{q}\left(N ; \varepsilon_{1}, \ldots, \varepsilon_{N}\right)\right), F u n\left(O_{q}(N\right.$; $\left.\left.\varepsilon_{1}, \ldots, \varepsilon_{N}\right)\right)$ and $\operatorname{Fun}\left(\operatorname{Sp} p_{q}\left(n ; \varepsilon_{1}, \ldots, \varepsilon_{2 n}\right)\right)$ of $[\mathrm{RTF}]$ (for $q>0$ ). Such construction can be done for each coquasitriangular (CQT) $*$-Hopf algebra $A$. The obtained object $\mathbb{A}^{\mathbb{C} R}$ is also a CQT $*$-Hopf algebra. We describe the theory of corepresentations of $\mathbf{A}^{\mathbb{C R}}$ in terms of such a theory for $\mathbf{A}$.
\end{abstract}

\section{§0. Introduction}

Quantum groups have recently attracted the attention of many physicists and mathematicians due to their remarkable properties. Some of these properties are similar to those of usual groups, while another properties, reflecting noncommutativity of the corresponding algebras, are suitable only in the "quantum' case. The theory is developed in two approaches. One of them ([Dr], $[\mathrm{J}])$ mainly deals with quantum universal enveloping algebras, while the second ([W1]-[W3], [Dr], [RTF]) investigates algebras of functions on quantum groups. In the present paper we follow the second approach. Noncommutative algebras which we consider here are usually $*$-Hopf algebras (sometimes we consider $C^{*}$-structure on them, which makes the underlying objects topological quantum groups), so we mainly deal with 'quantum algebraic real groups'.

Simple examples of such objects are the quantum $S U_{q}(2)$ groups (they are described in [W1], where the topological formulation is also given). Omitting the *-structure in the corresponding algebras we obtain the algebras of holomorphic polynomials on the complex quantum $S L_{q}(2)$ groups. But the problem

Communicated by H. Araki, May 7, 1991.

1991 Mathematics Subject Classification: 16W30, 20N99

* RIMS, Kyoto University, Kitashirakawa, Sakyo-ku, Kyoto 606, Japan.

On leave from: Department of Mathematical Methods in Physics, Faculty of Physics, University of Warsaw, Hoża 74, PL-00-682 Warszawa, Poland. 
of finding all polynomials on real quantum groups $S L_{q}(2, \mathbb{C})$ (and commutation relations between them) is not trivial. Its solution is given (for $q \in$ $(-1,1) \backslash\{0\})$ in $\left[\mathrm{PW}\right.$ (cf. also [CSSW]). The *-algebra of $S L_{q}(2, \mathbb{C})$ contains holomorphic polynomials and antiholomorphic polynomials. Commutation relations between them are also provided. It became "folk" knowledge (cf. $[\mathrm{Pu}]$ ) that the matrix which gives them is in fact (proportional to) the $\mathbb{R}$-matrix corresponding to $S U_{q}(2)$ (cf. Remark 7 of Section 3).

A natural problem arises: can we find real structures on all complex quantum groups $S L_{q}(N), O_{q}(N)$ (denoted in $[R T F]$ by $S O_{q}(N)$ ) and $S p_{q}(n)$, which were presented in $[\mathrm{RTF}]$ ? Is there any general construction, which, when applied to real quantum $S U_{q}(2)$ groups, yields quantum $S L_{q}(2, \mathbb{C})$ groups?

An interesting solution of the last problem was given in [Ko]. It is assumed there that we have a $*$-Hopf algebra $\mathbb{A}=(A, *, \phi, \kappa, e)$ with

a) unitary corepresentation $u$ such that its matrix elements generate $A$ as an algebra

b) selfadjoint matrix $R$ intertwining the second tensor power of $u$ with itself.

Then T. H. Koornwinder constructs a $*$-Hopf algebra $B$ as free product of Hopf algebra $\mathbb{A}$ and the conjugate Hopf algebra $\mathbb{A}^{j}$ with some relations (cf remark 2 after Thm 3.3). In the case where $A$ is the algebra of polynomials on $S U_{q}(2), B$ is (for some choice of $R$ ) the algebra of polynomials on real quantum group $S L_{q}(2, \mathbb{C}), q \in(-1,1) \backslash\{0\}$. Unfortunately, this procedure doesn't control the 'size' of $B$ (choice of $R$ is not unique).

The aim of the present paper is to solve our problem in a way which would give full information about the obtained object, expressed in terms of the initial quantum group. The main result is presented in Section 3. There we define the real complexification $\mathbb{A}^{\mathbb{C}}$ of $*-H o p f$ algebra $\mathbb{A}$ as a vector space $A \otimes A^{j}$ with $*$-Hopf structure given in terms of a given functional $\mathscr{R}$ on $A \otimes A$. More precisely, $\mathbb{A}^{\mathbb{C R}}$ is a $*$-Hopf algebra and we have an embedding $G \subset G^{\mathbb{C R}}$ of corresponding quantum groups, but under the assumption that the pair $(\mathbf{A}, \mathscr{R})$ is a coquasitriangular $(\mathrm{CQT}) *$-Hopf algebra. In a special case our construction and construction of [Ko] coincide (see remark 2 after Thm 3.3).

CQT Hopf algebras were investigated in [Ha], [LT] (cf. also earlier papers [Lyu], [Dr]). CQT Hopf algebras can be viewed as 'almost commutative' Hopf algebras. In Section 1 of this paper we define a CQT $*-H o p f$ algebra as a CQT Hopf algebra $(\mathbb{A}, \mathscr{R})$ with compatible $*$-structure. Next we present the relation between such objects and corresponding hermitian braidings, which are families of $R$-matrices (cf. [Lyu], [LT] in the case without *). Using it, we can prove Theorem 1.4, which allows us to provide examples of $\mathrm{CQT}$ *-Hopf algebras. For a given algebra defined by generators and relations (which express the existence of intertwiners between tensor powers of the 
fundamental matrix of generators) we formulate purely arithmetic conditions under which such algebras can be endowed with (CQT)(*-)Hopf or $C^{*}$ algebraic structure. Among conditions, the braid equation and compatibility of its solution with relations defining the algebra play the fundamental role. The proof uses methods which were presented (in the case of $S U_{q}(N)$ ) in [W3] and [W4].

In Section 2 we prove that the *-Hopf algebras $F u n\left(S U_{q}\left(N ; \varepsilon_{1}, \ldots, \varepsilon_{N}\right)\right)$, $\operatorname{Fun}\left(O_{q}\left(N ; \varepsilon_{1}, \ldots, \varepsilon_{N}\right)\right)$ and $\operatorname{Fun}\left(S p_{q}\left(n ; \varepsilon_{1}, \ldots, \varepsilon_{2 n}\right)\right)$ of $[\mathrm{RTF}]\left(q>0\right.$; for $S L_{q}$ with odd $N, O_{q}$ with even $N$ and $S p_{q}$ we can also put $q<0$ ) have a natural CQT *-Hopf algebra structure related to the corresponding solutions of braid equation (similar results, without $*$, were given in $[\mathrm{Dr}],[\mathrm{Ha}]$; nevertheless we provide an alternative proof which uses Thm 1.4 and shows that existence of all considered structures follows immediately from the form of assumed relations). Moreover, if $\varepsilon_{1}=\cdots=\varepsilon_{N}=1$, we describe topological structures on the underlying quantum groups; in the case of $S U_{q}$ we have then topological quantum groups $S_{q} U(N)$ of [W3].

In Section 3 we describe real complexifications of the above examples obtaining real quantum groups $S L_{q}\left(N, \mathbb{C} ; \varepsilon_{1}, \ldots, \varepsilon_{N}\right), O_{q}\left(N, \mathbb{C} ; \varepsilon_{1}, \ldots, \varepsilon_{N}\right)$, $S p_{q}\left(n, \mathbb{C} ; \varepsilon_{1}, \ldots, \varepsilon_{2 n}\right)$ (for $q=1$ they don't depend on the choice of $\varepsilon_{1}, \ldots, \varepsilon_{N}$; in this case we obtain defining relations of $S L(N, \mathbb{C}), O(N, \mathbb{C})$ and $S p(n, \mathbb{C})$ ). In our examples all corepresentations of the initial Hopf algebra $\mathbb{A}$ are completely reducible. In each such situation $\mathbb{A}^{\mathbb{C R}}$ has the same property. Moreover, irreducible corepresentations $w^{\alpha, \beta}$ of $\mathbb{A}^{\mathbb{R}}$ are numbered by pairs $\left(u^{\alpha}, u^{\beta}\right)$ of irreducible corepresentations of $\mathbb{A}$ (if one identifies objects, which are equivalent). Dimensions of $w^{\alpha, \beta}$, the objects conjugate to them and multiplicities in decompositions of tensor products of $w^{\alpha, \beta}$ are expressed in terms of the similar data for A. In this way we describe representation theory of quantum group $G^{\mathbb{C R}}$ (or, in other words, the theory of 'real representations of the complexification of $G^{\prime}$ ), where $G, G^{\mathbb{R}}$ are underlying objects of $\mathbb{A}, \mathbb{A}^{\mathbb{R}}$.

Furthermore, for each CQT *-Hopf algebra $(\mathbb{A}, \mathscr{R}), \mathbb{A}^{\mathbb{C R}}$ can be also endowed with a canonical CQT $*$-Hopf algebra structure. Thus we obtain an infinite chain of CQT *-Hopf algebras. Notice that $*$-structure plays an important role in that construction. In the Appendix we provide basic notions and facts concerning (*-)Hopf algebras.

According to [PW], there is a link between $F u n\left(S U_{q}(2)\right)^{\mathbb{C R}}$ and double group of $S U_{q}(2), q \in(0,1)$. It occurs that a similar situation takes place for any CQT $*$-Hopf algebra such that all its corepresentations are completely reducible (in fact we got the definition of $\mathbb{A}^{\mathbb{C}}$ using that link). We are going to present this material in a separate publication $[\mathrm{P}]$.

Throughout the paper we use the following notation. $W^{\prime}$ denotes the dual vector space of a vector space $W$. If $\alpha: W \rightarrow V$ is a linear mapping of vector spaces then $\alpha^{\prime}: V^{\prime} \rightarrow W^{\prime}$ denotes the dual mapping of $\alpha$. If $\alpha^{\prime}: V^{\prime} \rightarrow W^{\prime}$ 
and $\beta^{\prime}: X^{\prime} \rightarrow Y^{\prime}$ are dual linear mappings then we define their extended tensor product by formula

$$
\alpha^{\prime} \hat{\otimes} \beta^{\prime}=(\alpha \otimes \beta)^{\prime}:(V \otimes X)^{\prime} \rightarrow(W \otimes Y)^{\prime}
$$

(note that $\alpha^{\prime}, \beta^{\prime}$ determine $\alpha, \beta$ uniquely). The same definition works also for antilinear dual mappings. (If $\alpha: W \rightarrow V$ is antilinear then the antilinear dual mapping of $\alpha, \alpha^{\prime}: V^{\prime} \rightarrow \mathbb{W}^{\prime}$, is given by $\left.\left[\alpha^{\prime}(\eta)\right](w)=\overline{\eta(\alpha(w))}, \eta \in V^{\prime}, w \in W\right)$.

If $V, W$ are vector spaces, then $s=s_{V W}: V \otimes W \rightarrow W \otimes V$ is a linear map such that

$$
s(x \otimes y)=y \otimes x
$$

$x \in \mathbb{V}, y \in W$.

Let $A$ be a vector space and $v, w$ matrices with entries in $A: v \in M_{m}(A)$, $w \in M_{n}(A), m, n \in \mathbb{N}$. Then we define $v \otimes w \in M_{m n}(A \otimes A)$ by

$$
(v \otimes w)_{i j, k l}=v_{i k} \otimes w_{j l},
$$

$i, k=1, \ldots, m ; j, l=1, \ldots, n$. If $A$ is an algebra then we have also the tensor product $v$ (1) $w \in \mathbb{M}_{m n}(A)$ :

$$
(v(1) w)_{i j, k l}=v_{i k} w_{j l}
$$

$i, k=1, \ldots, m ; j, l=1, \ldots, n$. We set $v^{\oplus l}=v(1) \cdots(1) v(l$ times, if $A$ has unity $I$ then $\left.v^{\oplus 0}=(I)\right)$. Moreover, let $A$ be a $*$-algebra. Then matrix $\bar{v}$ conjugate to $v$ is defined by

$$
\bar{v}_{i j}=v_{i j}^{*}, \quad i, j=1, \ldots, m \text {. }
$$

We have

$$
\overline{v \oplus w}=s_{v w}^{-1}(\bar{w} \oplus \bar{v}) s_{v w}
$$

We denote $v^{*}{ }_{i j}=v_{j i}{ }^{*}, i, j=1, \ldots, m$. If $S: X \rightarrow Y$ is a linear mapping of vector spaces then formula

$$
\left[S^{\otimes i d}(v)\right]_{i j}=S\left(v_{i j}\right),
$$

$i, j=1, \ldots, m, v=\left(v_{i j}\right)_{i, j=1}^{m} \in M_{m}(X)$, defines a linear mapping $S^{\otimes i d}: M_{m}(X) \rightarrow$ $M_{m}(Y), m \in \mathbb{N}$.

Let $\psi \in\left(\mathbb{C}^{k}\right)^{\otimes r}, r \geq 2$. Then by using the canonical basis in $\mathbb{C}^{k}$ denoted by $e_{1}, \ldots, e_{k}$ we obtain

$$
\psi=\sum_{i_{1}, \ldots, i_{r}=1}^{k} \psi_{i_{1} \ldots i_{r}} e_{i_{1}} \otimes \cdots \otimes e_{i_{r}},
$$

$\psi_{i_{1} \ldots i_{r}} \in \mathbb{C}$. We define arrays $\psi_{i}^{-}=\left(\psi_{i i_{2} \ldots i_{r}}\right)_{i_{2}, \ldots, i_{r}=1}^{k}, \psi_{i}^{+}=\left(\psi_{i_{1} \ldots i_{r-1}}\right)_{i_{1}, \ldots, i_{r-1}=1}^{k}$, $i=1, \ldots, k$. If $\psi_{i}^{-}\left(\psi_{i}^{+}\right.$, resp. $), i=1, \ldots, k$, are linearly independent, then we say that $\psi$ is left (right, resp.) nondegenerate (see [W3]). If both properties are satisfied, we say that $\psi$ is nondegenerate. In that context we identify a linear mapping $L \in \mathbb{L i n}\left(\mathbb{C},\left(\mathbb{C}^{k}\right)^{\otimes r}\right)$ with $L(1) \in\left(\mathbb{C}^{k}\right)^{\otimes r}$.

We always include 0 into the set $\mathbb{N}$ of natural numbers. 


\section{§1. Coquasitriangular *-Hopf Algebras}

In this Section we introduce and investigate the notion of coquasitriangular (CQT) *-Hopf algebra. In the following we use definitions and facts given in the Appendix.

Definition 1.1 (cf. [Dr], [Ha], [LT]). We say that $(\mathbb{A}, \mathscr{R})$ is a CQT*-Hopf algebra if

1) A is a *-Hopf algebra

2) $\mathscr{R}$ is an element of the algebra $A_{2}{ }^{\prime}=(A \otimes A)^{\prime}$ such that

$$
\begin{gathered}
\mathscr{R} \text { is invertible, } \\
(\Delta \hat{\otimes} i d) \mathscr{R}=\mathscr{R}_{13} \mathscr{R}_{23}, \\
(\text { id } \hat{\otimes} \Delta) \mathscr{R}=\mathscr{R}_{13} \mathscr{R}_{12}, \\
\sigma \Delta(a)=\mathscr{R} \cdot \Delta(a) \cdot \mathscr{R}^{-1}, \quad a \in A^{\prime}, \\
\sim \circ \mathscr{R} \circ *_{2}=\mathscr{R}
\end{gathered}
$$

$(\sim$ denotes complex conjugation in $\mathbb{C})$.

Omitting condition (1.4) we obtain the definition of $*$-Hopf algebra A with compatible hermitian element $\mathscr{R}$. Omitting the $*$-structure and (1.5) we get the definition of CQT Hopf algebra and Hopf algebra with compatible element $\mathscr{R}$ (if we also omit (1.4)).

Remark 1. Quasitriangular Hopf algebras were introduced in [Dr], while CQT Hopf algebras were investigated in [Ha], [LT] (neither paper uses *).

Remark 2. We can also consider a wider class of objects, with (1.5) replaced by $\sim \circ \mathscr{R} \circ *_{2}=\lambda \cdot \mathscr{R}$ for some $\lambda$ in the center of $A_{2}^{\prime}$. For clarity of exposition we put $\lambda=1$.

Remark 3. For the general $*$-Hopf algebra $\mathbb{A}$ there is no $\mathscr{R}$ satisfying the conditions of the above Definition. If such $\mathscr{R}$ exists, it is not unique, in general.

Remark 4. According to (1.4) $A$ is commutative iff $\mathscr{R}=1$ is admissible ( $A^{\prime}$ is 'cocommutative' in this case). Therefore CQT *-Hopf algebras can be also called quasicommutative $*$-Hopf algebras (due to (1.4) $\mathscr{R}$ describes 'noncommutativity' of $A$ ).

The notion of CQT *-Hopf algebra is related with the following

Definition 1.2 (cf. [Lyu], [LT]). Let $\mathbb{A}$ be a *-Hopf algebra. We say that a family $\left\{R^{v w}\right\}_{v, w \in R e p \mathbb{A}}$ is a hermitian braiding of $\mathbb{A}$ iff

$$
R^{v w} \in \operatorname{Lin}\left(\mathbb{C}^{\operatorname{dim} v} \otimes \mathbb{C}^{\operatorname{dim} w}, \mathbb{C}^{\operatorname{dim} w} \otimes \mathbb{C}^{\operatorname{dim} v}\right),
$$




$$
\begin{gathered}
(i d \otimes S) R^{v w}=R^{v^{\prime} w}(S \otimes i d) \quad \text { if } \quad S \in M o r\left(v, v^{\prime}\right), \\
(S \otimes i d) R^{v w}=R^{v w^{\prime}}(i d \otimes S) \quad \text { if } \quad S \in \operatorname{Mor}\left(w, w^{\prime}\right), \\
R^{v w} \text { is invertible, } \\
\left(R^{v w} \otimes i d\right)\left(i d \otimes R^{v^{\prime} w}\right)=R^{v \oplus v^{\prime}, w}, \\
\left(i d \otimes R^{v w^{\prime}}\right)\left(R^{v w} \otimes i d\right)=R^{v, w \oplus w^{\prime}}, \\
R^{v w} \in M o r(v \circledast w, w \oplus v), \\
\overline{s R^{\overline{v w}} S=R^{w v},}
\end{gathered}
$$

$v, w, v^{\prime}, w^{\prime} \in \operatorname{Rep} \mathbf{A}($ see (0.2)). Omitting the *-structure and condition (1.13) we get the definition of a Hopf algebra with braiding. Omitting condition (1.12) we obtain the definition of a *-Hopf algebra with compatible hermitian family. Omitting the *-structure and both conditions we have a Hopf algebra with compatible family.

Remark 5. Inserting $S=R^{v w} \in \operatorname{Mor}(v(1) w, w(1) v)$ into (1.7) and using (1.10) we obtain the braid relation

$$
\left(i d \otimes R^{v w}\right)\left(R^{v z} \otimes i d\right)\left(i d \otimes R^{w z}\right)=\left(R^{w z} \otimes i d\right)\left(i d \otimes R^{v z}\right)\left(R^{v w} \otimes i d\right)
$$

Remark 6. We will also investigate the condition

$$
R^{1 v}=i d
$$

where $v \in \operatorname{Rep} \mathbf{A}$ and $1=(I)$ is the trivial corepresentation.

The relation between the above two notions is given by

Proposition 1.3 (cf. [Lyu], [LT], [Ma]).

1) Let $\mathbf{A}$ be a Hopf algebra and $\mathscr{R} \in(A \otimes A)^{\prime}$. For each $v, w \in \operatorname{Rep} \mathbf{A}$ we define $\left(R^{v w}\right)_{i j, k l}=\mathscr{R}\left(v_{j k} \otimes w_{i l}\right), \quad j, k=1, \ldots, \operatorname{dim} v, \quad i, l=1, \ldots, \operatorname{dim} w$.

Then $\left\{R^{v w}\right\}_{v, w \in R e p \text { A }}$ satisfies (1.6)-(1.8). Let L be a sufficient set of corepresentations of $\mathbf{A}$. Then

a) $\mathscr{R}$ satisfies (1.2)-(1.3) iff $\left\{R^{v w}\right\}_{v, w \in L}$ satisfies (1.10)-(1.11)

b) The following are equivalent

i) $\mathscr{R}$ is a compatible element

ii) $\left\{R^{v w}\right\}_{v, w \in L}$ satisfies (1.9)-(1.11)

iii) $\left\{R^{v w}\right\}_{v, w \in L}$ satisfies (1.15), (1.10)-(1.11)

Each of the above conditions implies

$$
\begin{aligned}
\mathscr{R}^{-1}\left(v_{i l} \otimes w_{j k}\right) & =\left(R^{v w}\right)_{i j, k l}^{-1}, \\
R^{v 1} & =i d,
\end{aligned}
$$




$$
\begin{aligned}
\left(R^{v w}\right)_{i j, k l}^{-1} & =\left(R^{v^{c} w}\right)_{j l, i k}, \\
\left(R^{v w^{c}}\right)_{i j, k l}^{-1} & =\left(R^{v w}\right)_{k i, l j}, \\
R_{j l, i k}^{v^{c} w^{c}} & =R_{k i, l j}^{v w},
\end{aligned}
$$

$v, w \in \operatorname{Rep} \mathbf{A}, i, l=1, \ldots, \operatorname{dim} v ; j, k=1, \ldots, \operatorname{dim} w$.

c) (A, $\mathscr{R})$ is a CQT Hopf algebra iff $\left\{R^{v w}\right\}_{v, w \in L}$ satisfies (1.9)-(1.12)

d) Moreover, suppose that $\mathbf{A}$ is a *-Hopf algebra. Then

i) $(\mathbf{A}, \mathscr{R})$ is a $*$-Hopf algebra with compatible hermitian element iff $\left\{R^{v w}\right\}_{v, w \in L}$ satisfies (1.9)-(1.11) and (1.13)

ii) $(\mathbf{A}, \mathscr{R})$ is a CQT *-Hopf algebra iff $\left\{\boldsymbol{R}^{v w}\right\}_{v, w \in L}$ satisfies (1.9)-(1.13)

2) Let $\mathbf{A}$ be a CR Hopf algebra and $L$ be a sufficient set of corepresentations of $\mathbf{A}$.

a) Suppose that each of two families $\left\{R^{v w}\right\}_{v, w \in R e p \mathbf{A}},\left\{S^{v w}\right\}_{v, w \in R e p \mathbf{A}}$ satisfies (1.6)-(1.8). If $R^{v w}=S^{v w}$ for $v, w \in L$ then $R^{v w}=S^{v w}$ for $v, w \in R e p \mathbf{A}$.

b) Assume that the family $\left\{R^{v w}\right\}_{v, w \in L}$ satisfies (1.6)-(1.8). Then there exists a unique $\mathscr{R} \in(A \otimes A)^{\prime}$ such that (1.16) holds for $v, w \in L$.

In particular, for CR (*-) Hopf algebras, CQT (*-) Hopf algebra structures (compatible (hermitian) elements, resp.) are in one to one correspondence (given by (1.16)) with (hermitian) braidings (compatible (hermitian) families, resp.).

\section{Proof.}

1) Equation (1.16) implies (1.6)-(1.8).

a) Applying both sides of (1.2) to $v_{i l} \otimes v_{j k}^{\prime} \otimes w_{m n}$, we see that (1.2) is equivalent to (1.10) for $v, v^{\prime}, w \in L$. Similarly (1.3) is equivalent to (1.11) for $v, w, w^{\prime} \in L$.

b) Due to a), we may assume that in each case (1.10)-(1.11) hold for all $v, v^{\prime}, w, w^{\prime} \in \operatorname{Rep} \mathbf{A} . \quad$ Assume i). We set $\left(\tilde{R}^{v w}\right)_{i j, k l}=\mathscr{R}^{-1}\left(v_{i l} \otimes w_{j k}\right), i, l=1$, $\ldots, \operatorname{dim} v, j, k=1, \ldots, \operatorname{dim} w, v, w \in \operatorname{Rep} \mathbf{A}$. Then the equality $\mathscr{R}^{-1} \cdot \mathscr{R}=$ $\mathscr{R} \cdot \mathscr{R}^{-1}=I_{A_{2}}$ applied to $v_{i l} \otimes w_{j k}$ yields $\tilde{R}^{v w}=\left(R^{v w}\right)^{-1}$. So (1.17) and ii) hold. If ii) holds then setting $v=v^{\prime}=1, w \in L$ in (1.10) and using (1.9) we get (1.15) for $v \in L$ and iii) is satisfied. Assume iii). Then due to $(1.16) \mathscr{R}(I \otimes x)=e(x)$, $x \in A$, and (1.15) holds for all $v \in \operatorname{Rep} \mathbf{A}$. Set $D_{i j, 1}^{v}=E_{1, i j}^{v}=\delta_{i j}, i, j=1, \ldots$, $\operatorname{dim} v, v \in \operatorname{Rep} \mathbf{A}$. We have

$$
\begin{gathered}
D^{v} \in \operatorname{Mor}\left(1, v \oplus v^{c}\right), \\
E^{v} \in \operatorname{Mor}\left(v^{c} \oplus v, 1\right), \\
\left(i d \otimes E^{v}\right)\left(D^{v} \otimes i d\right)=i d, \\
\left(E^{v} \otimes i d\right)\left(i d \otimes D^{v}\right)=i d .
\end{gathered}
$$

For $v, w \in \operatorname{Rep} \mathbf{A}$ we put 


$$
\begin{aligned}
& B=\left(E^{w} \otimes i d \otimes i d\right)\left(i d \otimes R^{v w} \otimes i d\right)\left(i d \otimes i d \otimes D^{w}\right), \\
& C=\left(i d \otimes i d \otimes E^{v}\right)\left(i d \otimes R^{v^{c} w} \otimes i d\right)\left(D^{v} \otimes i d \otimes i d\right) .
\end{aligned}
$$

Then using (1.10), (1.22), (1.7) for $S=D^{v},(1.15)$ and (1.23), we obtain $R^{v w} C=i d$. Therefore $\left(R^{v w}\right)^{-1}=C$, which gives (1.19). Setting $w^{\prime}=1$ in (1.11) and using invertibility of $R^{v w}$, we get (1.18). In virtue of (1.11), (1.22), (1.8) for $S=D^{w}$, (1.18) and (1.24) one has $R^{v w^{c}} B=i d$. Hence $\left(R^{v w^{c}}\right)^{-1}=B$ and (1.20) follows. Comparing (1.19) (with $w$ replaced by $w^{c}$ ) and (1.20), we get (1.21). Put $\mathscr{R}^{\prime}=$ $\mathscr{R}(\kappa \otimes i d)$. Then

$$
\mathscr{R}^{\prime}\left(v_{i j} \otimes w_{k l}\right)=\mathscr{R}\left(\left(v^{c}\right)_{j i} \otimes w_{k l}\right)=\left(R^{v^{c} w}\right)_{k j, i l}=\left(R^{v w}\right)_{i k, l j}^{-1}
$$

(we used (1.19)). Therefore $\mathscr{R} \cdot \mathscr{R}^{\prime}=\mathscr{R}^{\prime} \cdot \mathscr{R}=1_{A_{2^{\prime}}}$ (we check it on $v_{i j} \otimes w_{k l}$ ), $\mathscr{R}$ is invertible and then i) follows.

c) According to b), in each case $\mathscr{R}$ is invertible and $\left(R^{v w}\right)^{-1}$ exist, $v$, $w \in L$. Applying both sides of (1.4) to $v_{i l} \otimes w_{j k}$ we get that (1.4) is a translation of

$$
a\left((w \oplus v)_{j i, k l}\right)=a\left(\left[R^{v w}(v \oplus w)\left(R^{v w}\right)^{-1}\right]_{j i, k l}\right), \quad a \in A^{\prime},
$$

which is equivalent to (1.12).

d) i) Applying both sides of (1.5) to $w_{j k} \otimes v_{i l}$ we get that (1.5) is equivalent to (1.13) for $v, w \in L$.

ii) follows from i) and c).

2) a) Let $v, w \in \operatorname{Rep} \mathbb{A}$. Then due to Prop. A.3

$$
I_{v}=\sum_{m \in D} a_{m} a_{m}^{\prime}, \quad I_{w}=\sum_{n \in E} b_{n} b_{n}^{\prime}
$$

for some $a_{m} \in \operatorname{Mor}\left(l_{m}, v\right), a_{m}^{\prime} \in \operatorname{Mor}\left(v, l_{m}\right), b_{n} \in \operatorname{Mor}\left(l_{n}^{\prime}, w\right), b_{n}^{\prime} \in \operatorname{Mor}\left(w, l_{n}^{\prime}\right), l_{m}, l_{n}^{\prime} \in$ $L, m \in D, n \in E$, where $D, E$ are finite sets. Using (1.7)-(1.8) we get

$$
\begin{aligned}
R^{v w} & =\sum_{m, n} R^{v w}\left(a_{m} a_{m}^{\prime} \otimes b_{n} b_{n}^{\prime}\right)=\sum_{m, n}\left(b_{n} \otimes a_{m}\right) R^{l_{m} l_{n}^{\prime}}\left(a_{m}^{\prime} \otimes b_{n}^{\prime}\right) \\
& =\sum_{m, n}\left(b_{n} \otimes a_{m}\right) S^{l_{m} l_{n}^{\prime}}\left(a_{m}^{\prime} \otimes b_{n}^{\prime}\right)=\sum_{m, n} S^{v w}\left(a_{m} a_{m}^{\prime} \otimes b_{n} b_{n}^{\prime}\right)=S^{v w} .
\end{aligned}
$$

b) Furthermore, suppose $z, t \in \operatorname{Rep} \mathbb{A}$ and

$$
I_{z}=\sum_{p \in F} c_{p} c_{p}^{\prime}, \quad I_{t}=\sum_{q \in G} d_{q} d_{q}^{\prime}
$$

for some $c_{p} \in \operatorname{Mor}\left(k_{p}, z\right), c_{p}^{\prime} \in \operatorname{Mor}\left(z, k_{p}\right), d_{q} \in \operatorname{Mor}\left(k_{q}^{\prime}, t\right), d_{q}^{\prime} \in \operatorname{Mor}\left(t, k_{q}^{\prime}\right), k_{p}, k_{q}^{\prime} \in$ $L, p \in F, q \in G$, where $F, G$ are finite sets. We set

$$
\begin{aligned}
& R_{(a b)}^{v w}=\sum_{m, n}\left(b_{n} \otimes a_{m}\right) R^{l_{m} l_{n}^{\prime}}\left(a_{m}^{\prime} \otimes b_{n}^{\prime}\right), \\
& R_{(c d)}^{z t}=\sum_{p, q}\left(d_{q} \otimes c_{p}\right) R^{k_{p} k_{q}^{\prime}}\left(c_{p}^{\prime} \otimes d_{q}^{\prime}\right) .
\end{aligned}
$$


Assume $S \in \operatorname{Mor}(v, z), \quad T \in \operatorname{Mor}(w, t)$. Then using (1.27), $d_{j}^{\prime} T b_{n} \in \operatorname{Mor}\left(l_{n}^{\prime}, k_{j}^{\prime}\right)$, $c_{i}^{\prime} S a_{m} \in \operatorname{Mor}\left(l_{m}, k_{i}\right)$, (1.25), (1.26), $c_{i}^{\prime} c_{p} \in \operatorname{Mor}\left(k_{p}, k_{i}\right), d_{j}^{\prime} d_{q} \in \operatorname{Mor}\left(k_{q}^{\prime}, k_{j}^{\prime}\right)$ and (1.28), we obtain

$$
\begin{aligned}
\left(d_{j}^{\prime} \otimes c_{i}^{\prime}\right)(T \otimes S) R_{(a b)}^{v w} & =\sum_{m, n}\left(d_{j}^{\prime} T b_{n} \otimes c_{i}^{\prime} S a_{m}\right) R^{l_{m} l_{n}^{\prime}}\left(a_{m}^{\prime} \otimes b_{n}^{\prime}\right) \\
& =\sum_{m, n} R^{k_{2} k_{j}^{\prime}}\left(c_{i}^{\prime} S a_{m} a_{m}^{\prime} \otimes d_{j}^{\prime} T b_{n} b_{n}^{\prime}\right) \\
& =\sum_{p, q} R^{k_{1} k_{j}^{\prime}}\left(c_{i}^{\prime} c_{p} c_{p}^{\prime} S \otimes d_{j}^{\prime} d_{q} d_{q}^{\prime} T\right) \\
& =\sum_{p, q}\left(d_{j}^{\prime} d_{q} \otimes c_{i}^{\prime} c_{p}\right) R^{k_{p} k_{q}^{\prime}}\left(c_{p}^{\prime} \otimes d_{q}^{\prime}\right)(S \otimes T) \\
& =\left(d_{j}^{\prime} \otimes c_{i}^{\prime}\right) R_{(c d)}^{z t}(S \otimes T) .
\end{aligned}
$$

Multiplying both sides from the left by $d_{j} \otimes c_{i}$ and summing over $i, j$, one gets

$$
(T \otimes S) R_{(a b)}^{v w}=R_{(c d)}^{z t}(S \otimes T)
$$

Specializing $v=z, w=t, S=i d, T=i d$, we see that $R_{(a b)}^{v w}$ doesn't depend on the choice of $a_{m}, a_{m}^{\prime}, b_{n}, b_{n}^{\prime}, l_{m}, l_{n}^{\prime}, D, E$. Put $R^{v w}=R_{(a b)}^{v w}$ (for $v, w \in L, R^{v w}$ are same as before). Then (1.6) holds and (1.29) implies (1.7)-(1.8).

We define $\mathscr{R} \in(A \otimes A)^{\prime}$ by (1.16) with $v, w \in I$ (cf. Prop. A.2). Let $\left\{S^{v w}\right\}_{v, w \in R e p \text { A }}$ be the family corresponding to $\mathscr{R}$ by (1.16). Obviously, $R^{v w}=$ $S^{v w}$ for $v, w \in I$, hence by a) $R^{v w}=S^{v w}$ for all $v, w \in R e p \mathbb{A}$. In particular, (1.16) holds for $v, w \in L$ and the desired element $\mathscr{R}$ exists. Notice that (1.16) $(v, w \in L)$ describes $\mathscr{R}$ on a set linearly generating $A \otimes A$. Therefore the desired element $\mathscr{R}$ is unique.

The following result allows us to construct $(*-)$ Hopf algebras, CQT (*-)Hopf algebras and compact matrix quantum groups. In the case of quantum $S U(N)$ groups, it is essentially contained in [W3], [W4].

Theorem 1.4. Let $H=\mathbb{C}^{N}$ (with canonical Hilbert space structure), $N>1$. We put $H^{k}=H \otimes \cdots \otimes H$ ( $k$ times), $k \in \mathbb{N}$. Suppose there are $C_{m} \in \operatorname{Lin}\left(H^{s_{m}}, H^{r_{m}}\right)$, $s_{m}, r_{m} \in \mathbb{N}, m=1, \ldots, P, P \in \mathbb{N}$.

We define $A$ as the algebra with unity $I$, generated by $u_{i j}, i, j=1, \ldots, N$, satisfying relations

$$
C_{m} u^{s_{m}}=u^{r_{m}} C_{m}, \quad m=1, \ldots, P,
$$

where $u^{l}=u$ (1) $\cdots$ (1) $u$ (l times), $u^{0}=(I), u=\left(u_{i j}\right)_{i, j=1}^{N} \in M_{N}(A)($ in (1.30) we treat $C_{m}$ as matrices).

Set $C_{0}=i d_{H}$. Tensor products of any number of elements $C_{m}, m=0,1$, ..., $P$, are called elementary monomials. Products of any number of elementary monomials (if their composition makes sense) are called monomials. We define $M\left(u^{k}, u^{l}\right)$ as the linear span of monomials belonging to $\operatorname{Lin}\left(H^{k}, H^{l}\right), k, l \in \mathbb{N}$. Let 
$B=B^{*} \in G L(H)$. For any $C \in \operatorname{Lin}\left(H^{k}, H^{l}\right)$ we set

Obviously $\widetilde{\widetilde{C}}=C$.

$$
\widetilde{C}=\left(B^{\otimes k}\right)^{-1} C^{*}\left(B^{\otimes l}\right) \in \operatorname{Lin}\left(H^{l}, H^{k}\right) .
$$

1) Assume there exist left nondegenerate $E \in M\left(u^{0}, u^{a}\right)$ and right nondegenerate $E^{\prime} \in M\left(u^{b}, u^{0}\right)^{*}$ (for some $a, b \geq 2$ ). Then there is a unique Hopf algebra structure $\mathrm{A}$ on $A$ which makes $u$ a corepresentation.

2) Assume

$$
\begin{aligned}
& \text { there exists a nondegenerate } E \in M\left(u^{0}, u^{a}\right) \quad(\text { for some } a \geq 2), \\
& \qquad \widetilde{C}_{m} \in M\left(u^{r_{m}}, u^{s_{m}}\right), \quad m=1, \ldots, P .
\end{aligned}
$$

Then there is a unique *-Hopf algebra structure $\mathbb{A}$ on $A$ such that

$u$ is a corepresentation of $\mathbb{A}$,

$$
u^{*}=B u^{-1} B^{-1} \text {. }
$$

3) Assume (1.31)-(1.32) for $B=i d$. Let $S \in \operatorname{Lin}\left(H^{k}, H^{l}\right)$. Then

$$
S u^{k}=u^{l} S \quad \Leftrightarrow \quad S \in M\left(u^{k}, u^{l}\right) .
$$

4) Assume (1.31)-(1.32) for $B=i d$. Then there exists maximal $C^{*}$-seminorm $\|\cdot\|$ on the *-algebra $A$ (cf. 2)). It occurs that $\|\cdot\|$ is a norm. Let $\bar{A}$ be the $C^{*}$-algebra obtained by the completion of $A$ with respect to the norm $\|\cdot\|$. Obviously, $\bar{A}$ is the universal $C^{*}$-algebra with unity generated by $u_{i j}, i, j=$ $1, \ldots, N$, satisfying (1.30) and $u^{*} u=u u^{*}=1$. Moreover, $(\bar{A}, u)$ is a compact matrix quantum group ('pseudogroup') in the sense of [W2]. In particular, $\mathbb{A}$ (cf. 2)) is $C R$.

5) Assume (1.31)-(1.32) for $B=i d$. Let $R \in \operatorname{Lin}\left(H^{2}, H^{2}\right)$. We define $R_{(k)(l)} \in \operatorname{Lin}\left(H^{k} \otimes H^{l}, H^{l} \otimes H^{k}\right)$ as follows:

$$
\begin{aligned}
R_{(0)(k)} & =R_{(k)(0)}=i d_{H^{k}}, \quad k=0,1,2, \ldots, \\
R_{(1)(1)} & =R \\
R_{(k+1)(1)} & =\left(R \otimes i d_{H^{k}}\right)\left(i d_{H} \otimes R_{(k)(1)}\right), \quad k=1,2, \ldots, \\
R_{(k)(l+1)} & =\left(i d_{H^{l}} \otimes R_{(k)(1)}\right)\left(R_{(k)(l)} \otimes i d_{H}\right), \quad k, l=1,2, \ldots,
\end{aligned}
$$

Assume the following compatibility relations:

$$
\begin{aligned}
& \left(i d_{H} \otimes C_{m}\right) R_{\left(s_{m}\right)(1)}=R_{\left(r_{m}\right)(1)}\left(C_{m} \otimes i d_{H}\right), \\
& \left(C_{m} \otimes i d_{H}\right) R_{(1)\left(s_{m}\right)}=R_{(1)\left(r_{m}\right)}\left(i d_{H} \otimes C_{m}\right),
\end{aligned}
$$

$m=1, \ldots, P$. Then there exists on $A$ a unique structure $(\mathbb{A}, \mathscr{R})$ of Hopf algebra with compatible element $\mathscr{R}$, satisfying (1.33) and

$$
R^{u u}=R \text {, }
$$


where $\left\{\boldsymbol{R}^{v w}\right\}_{v, w \in R e p \text { A }}$ is the compatible family corresponding to $\mathscr{R}$. Moreover, if $R \in M\left(u^{2}, u^{2}\right)$ then $(\mathbf{A}, \mathscr{R})$ is a CQT Hopf algebra.

6) Assume (1.31)-(1.32) and let $R \in M\left(u^{2}, u^{2}\right)$ satisfy (1.36)-(1.37). If moreover

$$
\begin{gathered}
R^{*}=(B \otimes B) R\left(B^{-1} \otimes B^{-1}\right), \\
C_{m}^{*} \in M\left(u^{r_{m}}, u^{s_{m}}\right), \quad m=1, \ldots, P
\end{gathered}
$$

(for $B=i d,(1.40)$ coincides with (1.32)), then there is a unique CQT *-Hopf alge-

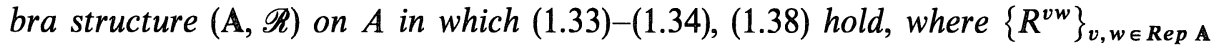
is the hermitian braiding corresponding to $\mathscr{R}$. Moreover, $\mathbf{A}$ is $C R$.

Remark 7. If $R \in M\left(u^{2}, u^{2}\right)$ then by (1.36)-(1.37) $R$ satisfies the braid relation

$(R \otimes i d)(i d \otimes R)(R \otimes i d)=(i d \otimes R)(R \otimes i d)(i d \otimes R)$.

Proof of Theorem 1.4. We sum over repeated indices (Einstein's convention). Let $w_{i j}=u_{i k} \otimes u_{k j}, i, j=1, \ldots, N$. Then the matrix $w=\left(w_{i j}\right)_{i, j=1}^{N}\left(1_{N \times N}\right.$, resp.) also satisfies (1.30) and there exists a unital homomorphism $\phi: A \rightarrow A \otimes A$ $\left(e: A \rightarrow \mathbb{C}\right.$, resp.) such that $\phi\left(u_{i j}\right)=u_{i k} \otimes u_{k j}\left(e\left(u_{i j}\right)=\delta_{i j}\right.$, resp.), $i, j=1, \ldots, N$. Consequently, (A.1)-(A.2) hold.

1) Since $E$ is left nondegenerate, there exists $W: H \rightarrow H^{a-1}$ such that $E_{i i_{2} \ldots i_{a}} W_{i_{2} \ldots i_{a}, k}=\delta_{i k}, i, k=1, \ldots, N$. Multiplying equality $u^{a} E=E u^{0}$, i.e.

$$
u_{i j} u_{i_{2} j_{2}} \ldots u_{i_{a} j_{a}} E_{j j_{2} \ldots j_{a}}=E_{i i_{2} \ldots i_{a}} I, \quad i, i_{2}, \ldots, i_{a}=1, \ldots, N,
$$

by $W_{i_{2} \ldots i_{a}, k}$ and summing over $i_{2}, \ldots, i_{a}$, we get that $u$ is right-invertible. Similarly, using $E^{\prime}$, one proves that $u$ is left-invertible. Thus $u^{-1}$ exists.

Let us notice that $\left(u^{s}\right)^{-1}=\left(u^{-1}\right)^{\Phi^{\prime} s}$ where $\mathbb{D}^{\prime}$ denotes tensor product with respect to the algebra $A^{o p}$ with opposite multiplication. Then (1.30) implies

$$
C_{m}\left(u^{-1}\right)^{\Phi^{\prime} s_{m}}=\left(u^{-1}\right)^{\Phi^{\prime} r_{m}} C_{m}, \quad m=1, \ldots, P,
$$

and hence $\left(u^{-1}\right)_{i j}$ satisfy (1.30) in $A^{o p}$. Consequently, there is a linear antimultiplicative unital mapping $\kappa: A \rightarrow A$ such that $\kappa\left(u_{i j}\right)=\left(u^{-1}\right)_{i j}, i, j=1, \ldots, N$. We obtain (A.3) for $x=u_{i j}$ and hence for all $x$. Thus the desired $\mathbf{A}$ exists. Uniqueness follows from the uniqueness of $\phi$.

2) According to (1.32), relations (1.30) imply

$$
\tilde{C}_{m} u^{r_{m}}=u^{s_{m}} \tilde{C}_{m}, \quad m=1, \ldots, P .
$$

Define $\tilde{M}\left(u^{k}, u^{l}\right)$ as the sets of combinations of products of tensor products of $\tilde{C}_{0}=i d_{H}, \tilde{C}_{1}, \ldots, \tilde{C}_{P}$. By (1.32),

$$
C_{m}=\tilde{\widetilde{C}}_{m} \in \tilde{M}\left(u^{s_{m}}, u^{r_{m}}\right), \quad m=1, \ldots, P .
$$

Therefore (1.43) imply (1.30) and both sets of relations are equivalent. 
$E \in M\left(u^{0}, u^{a}\right)$ is left nondegenerate, while $\left(B^{\otimes a}\right) E=\tilde{E}^{*} \in M\left(u^{a}, u^{0}\right)^{*}$ is right nondegenerate. Thus by 1$)$ it suffices to prove the existence of $*$-structure which satisfies (1.34) (equation (1.34) determines the mapping * uniquely on the generators).

Let $z=\left(u^{-1}\right)^{T}$ (the matrix transposed to $\left.u^{-1}\right), v=\left(B^{-1}\right)^{T} z \mathbb{B}^{T}$. Then (1.42) yields $z^{\Phi^{\prime} s_{m}} C_{m}^{T}=C_{m}^{T} z^{\Phi^{\prime} r_{m}}$ and $v^{\Phi^{\prime} s_{m}} \widetilde{\widetilde{C}}_{m}=\overline{\widetilde{C}}_{m} v^{\Phi^{\prime} r_{m}}$. Therefore $v_{i j}, i, j=1, \ldots$, $N$, satisfy, in the conjugate algebra $A^{j},(1.43)$ and thus also (1.30). So there exists an antilinear antimultiplicative unital mapping $*: A \rightarrow A$ such that $u_{i j}{ }^{*}=$ $v_{i j}, i, j=1, \ldots, N$. Hence $u^{*}=v^{T}=B u^{-1} B^{-1}$ and (1.34) holds. Consequently,

$$
u^{* *}=\left(B^{-1}\right)\left(u^{*}\right)^{-1} B=B^{-1} B u B^{-1} B=u
$$

and $*$ is an involution. Moreover $\bar{u}=\left(B^{-1}\right)^{T} u^{c} B^{T}$ is a corepresentation and hence $\phi *=(* \otimes *) \phi$ (it holds on $\left.u_{i j}, i, j=1, \ldots, N\right)$.

3) 4) We use the terminology and results of [W3]. We essentially repeat the reasoning of [W3], which leads to quantum $S U(N)$ groups.

Let $W=\left\{u^{l}: l \in \mathbb{N}\right\}$. Set $H_{u^{l}}=H^{l}, u^{k} \cdot u^{l}=u^{k} \mathbb{(} u^{l}\left(=u^{k+l}\right), k, l \in \mathbb{N}, f=u$. Then $=\left(W,\left\{H_{w}\right\}_{w \in W},\{M(v, w)\}_{v, w \in W}, \cdot, f\right)$ is a concrete monoidal $W^{*}$-category with distinguished object. Let $\mathbb{Z}=\left(\mathbb{Z},\left\{H_{w}\right\}_{w \in Z},\{M(v, w)\}_{v, w \in Z}, \cdot, f\right)$ be the completion of $\mathbb{W}$. By the argument of [W3, pages 60-61] we conclude that $f$ has a complex conjugation in $\mathbb{Z}$. Let $C$ be the universal $C^{*}$-algebra with unity generated by $\tilde{u}_{i j}, i, j=1, \ldots, N$, such that $\tilde{u}=\left(\tilde{u}_{i j}\right)_{i, j=1}^{N}$ is unitary and satisfies (1.30). Then $G=(B, \tilde{u})$ is universal $\mathbb{Z}$-admissible pair, $G$ is a compact matrix pseudogroup and for any $S \in \operatorname{Lin}\left(H^{k}, H^{l}\right)$

$$
S \tilde{u}^{k}=\tilde{u}^{l} S \Leftrightarrow S \in M\left(u^{k}, u^{l}\right)
$$

(use [W3, Thm 1.3 and Prop. 2.7.2]). Let $\mathscr{A}$ be the $*$-subalgebra considered in the proof of $[\mathrm{W} 3, \mathrm{Thm} 1.3]$ in the case of category $\mathbb{Z}$. Then the maximal $C^{*}$-seminorm on $\mathscr{A}$ exists. Moreover this seminorm is a norm and $C$ coincides with completion of $\mathscr{A}$ in that norm. According to [W3, page 59], $\mathscr{A}$ is the dense $*$-subalgebra (with unity) of $C$, generated by $\tilde{u}_{i j}$. Let us notice that $A$ is the universal $*$-algebra with unity generated by $u_{i j}, i, j=1, \ldots, N$, satisfying (1.30) and

$$
u^{*} u=u u^{*}=1_{M_{N}(A)},
$$

while $\mathscr{A}$ is a unital $*$-algebra generated by $\tilde{u}_{i j}$ satisfying (1.30) and (1.45). Therefore we have a unital $*$-epimorphism $\rho: A \rightarrow \mathscr{A}$ such that $\rho\left(u_{i j}\right)=\tilde{u}_{i j}$. In order to prove 3)4) it remains to check that $\rho$ is faithful (then we can identify $A$ with $\mathscr{A}, u$ with $\tilde{u}, \bar{A}$ with $C$ and (1.44) gives (1.35)). To this end we need

Definition 1.5 (cf. [W3], page 40). Let $\mathbb{R}=\left(R,\left\{H_{r}\right\}_{r \in R},\{M o r(r, s)\}_{r, s \in R}\right.$, $\cdot, f)$ be a concrete monoidal $W^{*}$-category with distinguished object. We say that $M=\left(B,\left\{v^{r}\right\}_{r \in R}\right)$ is an a-model of $\mathbb{R}$ if $B$ is a *-algebra with unity $I$ and 
$v^{r}$ are unitary elements of $B\left(H^{r}\right) \otimes B, r \in R$, such that

$$
\begin{gathered}
v^{r s}=v^{r} \oplus v^{s}, \\
v^{r}(t \otimes I)=(t \otimes I) v^{s},
\end{gathered}
$$

for any $r, s \in R$ and $t \in M o r(s, r)$.

Lemma 1.6 (cf. [W3, Prop. 2.7.5, Prop. 3.1.4, Prop. 3.3]). Let $\mathbf{R}, M$ be as in Def. 1.5 .

1) Let $\mathbf{S}$ be the completion of $\mathbb{R}$. Then $M$ has a unique extension to an a-model $N$ of $\mathbf{S}$.

2) Assume that $\mathbb{R}$ is complete, $\bar{f}$ exists and $\{f, \bar{f}\}$ generates $\mathbb{R}$. Let $(B, u)$ be the universal $\mathbf{R}$-admissible pair, $\left(B,\left\{u^{r}\right\}_{r \in R}\right)$ the corresponding model and $\mathscr{A}$ be the unital *-algebra generated by all $u_{i j}$. Then there exists a unital *homomorphism $\varphi_{M}: \mathscr{A} \rightarrow B$ such that $\left(\right.$ id $\left.\otimes \varphi_{M}\right) u^{r}=v^{r}, r \in R$.

Proof. The same as in [W3].

We return to the proof of Thm. 1.4. Clearly $M=\left(A,\{w\}_{w \in W}\right)$ is an a-model of $\mathbf{W}$. Thus by Lemma 1.6 there exists a unital $*$-homomorphism $\varphi_{M}: \mathscr{A} \rightarrow A$ such that $\varphi_{M}\left(\tilde{u}_{i j}\right)=u_{i j}, i, j=1, \ldots, N$. Thus $\varphi_{M} \rho=i d, \rho \varphi_{M}=i d$ and both $\rho, \varphi_{M}$ are $*$-isomorphisms. Therefore 3) 4) follow.

5) By 4), $\mathbf{A}$ is $\mathrm{CR}$. Since $u_{i j}$ generate $A$ as an algebra with unity, hence $L=\left\{u^{k}\right\}_{k \in \mathbb{N}}$ is sufficient (cf. the Appendix). But (1.38) implies $R^{u^{k} u^{l}}=R_{(k)(l)}$ and therefore (cf. Prop. 1.3.2. b) uniqueness of $\mathscr{R}$ follows.

Now we will prove the existence of $\mathscr{R}$. Due to (1.35)-(1.37), $\widetilde{R}^{u^{k_{u} l}}=R_{(k)(l)}$, $k, l \in \mathbb{N}$, satisfy (1.6)-(1.8). Therefore, by Prop. 1.3.2. b) there exists $\mathscr{R} \in(A \otimes A)^{\prime}$, whose corresponding family $\left\{R^{v w}\right\}_{v, w \in R e p A}$ satisfies $R^{v w}=\tilde{R}^{v w}, v, w \in L$. In particular $R^{u u}=R_{(1)(1)}=R$. It is straightforward that $\left\{R^{v w}\right\}_{v, w \in L}$ satisfy (1.10)(1.11) and (1.15). Then Prop. 1.3.1. b) implies that $\mathscr{R}$ is a compatible element. Moreover, if $R \in M\left(u^{2}, u^{2}\right)$ then by (1.35) $R \in \operatorname{Mor}\left(u^{2}, u^{2}\right)$ and $R^{u^{k} u^{l}}=R_{(k)(l)} \in$ $\operatorname{Mor}\left(u^{k}(1) u^{l}, u^{l}(1) u^{k}\right)$. Using Prop. 1.3.1. b) and c), (A, $\left.\mathscr{R}\right)$ is a CQT Hopf algebra.

6) Due to (1.40), equation (1.32) is satisfied also for $B=i d$. Uniqueness of (CQT)(*-)Hopf algebra structures on $A$ follows from 1) 2) 5). By 2) 5) there exist the desired $*$-Hopf algebra and CQT Hopf algebra structures on $A$ which give the same (by uniqueness) Hopf algebra structure A. By Prop. 1.3.1 it remains to check (1.13) for $v, w \in L$.

Denote $v=B u B^{-1}$. Then $\bar{u}=v^{c}$ and using (1.21)

$$
\overline{S R^{\bar{u} \bar{u}} S}=\overline{s R^{v^{c} v^{c}} S}=\left(R^{v v}\right)^{*} .
$$

But $B \in \operatorname{Mor}(u, v)$, hence $R^{v v}=(B \otimes B) R^{u u}\left(B^{-1} \otimes B^{-1}\right)$. Thus using (1.39),

$$
\overline{S R^{\bar{u} \bar{u}} S}=\left(B^{-1} \otimes B^{-1}\right)\left(R^{u u}\right) *(B \otimes B)=R^{u u}
$$


and (1.13) holds for $v=w=u$. Hence, taking into account (1.10)-(1.11), equation (1.13) holds for all $v, w \in L$.

\section{§2. Examples}

In this Section we prove that 'algebraic quantum groups' $S U_{q}\left(N ; \varepsilon_{1}, \ldots, \varepsilon_{N}\right)$, $O_{q}\left(N ; \varepsilon_{1}, \ldots, \varepsilon_{N}\right)$ and $S p_{q}\left(n ; \varepsilon_{1}, \ldots, \varepsilon_{2 n}\right)$ defined in $[\mathrm{RTF}]$ correspond (for $q>0$ ) to CQT *-Hopf algebras. Thus we illustrate the theory presented in the previous Section.

Remark 1. Similar results (but without $*$ and in more general context) were given in $[\mathrm{Dr}]$, [Ha]. Nevertheless we present here an alternative proof, which illustrates Thm. 1.4.

In the following, for fixed $N \in \mathbb{N}$, we set $H=\mathbb{C}^{N}, H^{k}=H^{\otimes k}, k=0,1, \ldots$.

Example 2.1 (cf. [W3], [RTF]). Let $N=n+1$ for the series $A_{n}, n=1$, $2, \ldots$ We define $A$ as the algebra with unity generated by $u_{i j}, i, j=1, \ldots$, $N$, satisfying

$$
\begin{gathered}
u^{\oplus N} E=E u^{\oplus 0}, \\
E^{*} u^{\oplus N}=u^{\oplus 0} E^{*}, \\
R(u \oplus u)=(u \oplus u) R,
\end{gathered}
$$

where $E \in \operatorname{Lin}\left(\mathbb{C}, H^{\otimes N}\right)$ and $R \in \operatorname{Lin}\left(H^{\otimes 2}, H^{\otimes 2}\right)$ are given by

$$
\begin{gathered}
E_{i_{1} \ldots i_{N}}= \begin{cases}0 & \text { for }\left\{i_{1}, \ldots, i_{N}\right\} \varsubsetneqq\{1, \ldots, N\} \\
(-q)^{\#\left\{\left(i_{k}, i_{l}\right): i_{k}>i_{l}, k<l\right\}} & \text { for }\left\{i_{1}, \ldots, i_{N}\right\}=\{1, \ldots, N\},\end{cases} \\
R=q^{-1 / N}\left[q \sum_{i=1}^{N}|i i\rangle\left\langle i i\left|+\sum_{\substack{i, j=1 \\
i \neq j}}^{N}\right| j i\right\rangle\left\langle i j\left|+\left(q-q^{-1}\right) \sum_{\substack{i, j=1 \\
i<j}}^{N}\right| i j\right\rangle\langle i j|\right],
\end{gathered}
$$

$u=\left(u_{i j}\right)_{i, j=1}^{N}, u^{\oplus 0}=(I), q>0$ (for $N$ odd, we also admit $q<0$ ).

Remark 2.

$$
R=q^{-1 / N} \hat{R}_{q}=q^{1-1 / N} \sigma_{W}^{-1}
$$

where $\hat{R}_{q}$ is given in Section 1.3 of [RTF] and $\sigma_{W}$ in (4.13) of [W3] (we set $\mu=q$ ). Moreover, due to [W3],

$$
\sigma_{W}=i d_{H^{2}}-\left(\prod_{k=1}^{N-2}\left(q^{2}+q^{4}+\cdots q^{2 k}\right)^{-1}\right)\left(i d_{H^{2}} \otimes E^{*}\right)\left(E \otimes i d_{H^{2}}\right)
$$

and therefore (2.3) is a consequence of (2.1)-(2.2).

Example 2.2 ([RTF]). Let $N=2 n+1$ for the series $B_{n}, N=2 n$ for the series $C_{n}$ and $D_{n}$. We define $A$ as the algebra with unity $I$, generated by $u_{i j}$, 
$i, j=1, \ldots, N$, satisfying

$$
\begin{aligned}
(u \oplus u) C & =C u^{\oplus 0}, \\
C^{*}(u \oplus u) & =u^{\oplus 0} C^{*}, \\
R(u \oplus u) & =(u \oplus u) R,
\end{aligned}
$$

where $C \in \operatorname{Lin}\left(\mathbb{C}, H^{\otimes 2}\right)$ and $R \in \operatorname{Lin}\left(H^{\otimes 2}, H^{\otimes 2}\right)$ are given by

$$
\begin{gathered}
C=\sum_{i=1}^{N} \theta_{i} q^{-\rho_{i}}\left|i i^{\prime}\right\rangle, \\
R=q \sum_{\substack{i=1 \\
i \neq i^{\prime}}}^{N}|i i\rangle\left\langle i i\left|+\sum_{\substack{i=1 \\
i=i^{\prime}}}^{N}\right| i i\right\rangle\left\langle i i\left|+\sum_{\substack{i, j=1 \\
j \neq i, i^{\prime}}}^{N}\right| j i\right\rangle\left\langle i j\left|+q^{-1} \sum_{\substack{i=1 \\
i \neq i^{\prime}}}^{N}\right| i i^{\prime}\right\rangle\left\langle i^{\prime} i\right| \\
+\left(q-q^{-1}\right) \sum_{\substack{i, j=1 \\
i<j}}^{N}|i j\rangle\left\langle i j\left|-\left(q-q^{-1}\right) \sum_{\substack{i, j=1 \\
i<j}}^{N} q^{\rho_{J}-\rho_{r}} \theta_{i} \theta_{j}\right| j^{\prime} j\right\rangle\left\langle i i^{\prime}\right|,
\end{gathered}
$$

$i^{\prime}=N+1-i, j^{\prime}=N+1-j, \theta_{i}=1, i=1, \ldots, N$, for the series $B_{n}$ and $D_{n}$, $\theta_{i}=1, i=1, \ldots, N / 2, \theta_{i}=-1, i=N / 2+1, \ldots, N$, for the series $C_{n}$ and

$$
\left(\rho_{1}, \ldots, \rho_{N}\right)=\left\{\begin{array}{r}
\left(n-\frac{1}{2}, n-\frac{3}{2}, \ldots, \frac{1}{2}, 0,-\frac{1}{2}, \ldots,-n+1 / 2\right) \text { for } B_{n} \\
(n, n-1, \ldots, 1,-1, \ldots,-n) \text { for } C_{n} \\
(n-1, n-2, \ldots, 1,0,0,-1, \ldots,-n+1) \text { for } D_{n}
\end{array}\right\},
$$

$u=\left(u_{i j}\right)_{i, j=1}^{N}, u^{\oplus 0}=(I), q \in \mathbb{R} \backslash\{0\}\left(q>0\right.$ for $\left.B_{n}\right)$.

Remark 3.

$$
R=\hat{R}_{q}
$$

where $\hat{R}_{q}$ is given in Section 1.4 of [RTF].

Proposition 2.3. Consider situation from Examples 2.1-2.2. Let $B=$ $\operatorname{diag}\left(\varepsilon_{1}, \ldots, \varepsilon_{N}\right), \varepsilon_{i}^{2}=1, i=1, \ldots, N$. In the case of series $B_{n}, C_{n}, D_{n}$ we assume $\varepsilon_{i^{\prime}}=\varepsilon_{i}, i=1, \ldots, N, \varepsilon_{i}=1$ for $i=i^{\prime}$. Then there exists a unique CQT*-Hopf algebra structure $(\mathbf{A}, \mathscr{R})$ on $A$ such that
a) $u$ is a corepresentation
b) $u^{*} B u=B, u B u^{*}=B$
c) $R^{u u}=R$

where $\left\{\boldsymbol{R}^{v w}\right\}_{v, w \in \boldsymbol{R e p} \mathbf{A}}$ is the hermitian braiding corresponding to $\mathscr{R}$. Moreover A is $C R$.

Proof. $\quad B=B^{*} \in G L(H)$. We shall check the assumptions of Thm. 1.4.6. First notice that $E$ (cf. [W3, page 63]) and $C$ are nondegenerate. Next,

$$
R^{*}=R,
$$


hence (1.40) follows. Moreover $\left(B^{\otimes N}\right) E=\varepsilon_{1} \ldots \varepsilon_{N} E,(B \otimes B) C=C$,

$$
(B \otimes B) R=R(B \otimes B)
$$

and also (1.32) holds. Combining (2.14) with (2.15), we get (1.39). By construction $R \in M\left(u^{2}, u^{2}\right)$. It remains to prove (1.36)-(1.37).

In the case of $A_{n}$ we need

$\left(R \otimes i d_{H}\right)\left(i d_{H} \otimes R\right)\left(R \otimes i d_{H}\right)=\left(i d_{H} \otimes R\right)\left(R \otimes i d_{H}\right)\left(i d_{H} \otimes R\right)$,

$$
\begin{gathered}
i d_{H} \otimes E=R_{(N)(1)}\left(E \otimes i d_{H}\right), \\
E \otimes i d_{H}=R_{(1)(N)}\left(i d_{H} \otimes E\right), \\
\left(i d_{H} \otimes E^{*}\right) R_{(N)(1)}=E^{*} \otimes i d_{H}, \\
\left(E^{*} \otimes i d_{H}\right) R_{(1)(N)}=i d_{H} \otimes E^{*} .
\end{gathered}
$$

By (2.6), equation (2.16) follows from (4.16) of [W3] (and also from [RTF]). Moreover, (4.34)-(4.35) of [W3] imply (2.18)-(2.19). Next, multiplying the last equation on page 69 of [W3] from left by (id $\left.\otimes E^{*}\right)$ and using (4.32)-(4.33) of [W3] we get (2.20). Its hermitian conjugation gives (2.17).

In the cases of $B_{n}, C_{n}$ and $D_{n}$ we need (2.16),

$$
\begin{aligned}
i d \otimes C & =(R \otimes i d)(i d \otimes R)(C \otimes i d), \\
C \otimes i d & =(i d \otimes R)(R \otimes i d)(i d \otimes C), \\
i d \otimes C^{*} & =\left(C^{*} \otimes i d\right)(i d \otimes R)(R \otimes i d), \\
C^{*} \otimes i d & =\left(i d \otimes C^{*}\right)(R \otimes i d)(i d \otimes R) .
\end{aligned}
$$

Due to (2.13) and [RTF], (2.16) holds. Next, we shall prove (2.21)-(2.22). According to $[\mathrm{RTF}], R-\mathbb{R}^{-1}=\left(q-q^{-1}\right)(I-K)$, where

$$
K=\theta C C^{*} \text {, }
$$

$\theta=1$ for $B_{n}$ and $D_{n}, \theta=-1$ for $C_{n}$. Hence, using $[\mathbb{R e}]$, we get

$(i d \otimes R)(R \otimes i d)(i d \otimes K)=(K \otimes i d)(i d \otimes K)$,

$(R \otimes i d)(i d \otimes R)(K \otimes i d)=(i d \otimes K)(K \otimes i d)$.

Using that, $(2.25),\left(C^{*} \otimes i d\right)(i d \otimes C)=\theta \cdot i d,\left(i d \otimes C^{*}\right)(C \otimes i d)=\theta \cdot i d$ and surjectivity of $C^{*}$ we get (2.21)-(2.22). Their hermitian conjugations give (2.23)(2.24).

Now using Thm. 1.4.6 we obtain the desired result.

Remark 4. Except $A_{n}$ with $N$ odd, we can replace $R$ by $(-R)$ and Prop. 2.3 still holds (by the same proof). 
Remark 5. Let us consider $A_{n}$ and admit all $q \in \mathbb{R} \backslash\{0\}$ and all $N$ values of $q^{-1 / N}$ in the expression for $R$. Then Prop. 2.3 still holds, but with the wider definition of CQT *-Hopf algebra, which was presented in Remark 2 after Def. 1.1 (braiding is now hermitian in some wider sense).

Remark 6. 1. Let $q \in \mathbb{R} \backslash\{0\}\left(q>0\right.$ for $\left.B_{n}\right)$. In the case of $A_{n}$ consider relations

$$
\begin{aligned}
& u^{\oplus N} E=E u^{\oplus 0}, \\
& u^{*} u=u u^{*}=1 .
\end{aligned}
$$

Let $\mathscr{A}_{0}$ be the $*$-algebra with unity generated by $(2.26)-(2.27)$ and $C\left(S_{q} U(N)\right)$ be the unital $C^{*}$-algebra generated by (2.26)-(2.27) (see [W3]). We claim that $\mathscr{A}_{0}$ is equal to $A$. First assume (2.26)-(2.27). Then (2.1)-(2.2) hold. By remark 2 after Ex.2.1 we also get (2.3). Conversely, assume (2.1)-(2.3). Then (2.26) is satisfied and putting $B=i d$ we obtain a $*$-structure on $A$, in which (2.27) holds. Therefore we can identify $A$ with $\mathscr{A}_{0}$ and (see [Ro]) with Fun $\left(S L_{q}(N)\right)$ of $[\mathrm{RTF}]$. Moreover, by Thm. 1.4 .4 (its assumptions have already been checked) the natural $*$-homomorphism $\rho$ from $A$ (with $*$ defined by (2.27)) into the unital $C^{*}$-algebra $\bar{A}$, generated by $(2.1)-(2.3)$ and $(2.27)$ is faithful (this is not generally true for an arbitrary set of relations). Similarly as before, we can identify $\bar{A}$ with $C\left(S_{q} U(N)\right)$. Thus

$$
A \cong \mathscr{A}_{0} \cong \mathscr{A}_{S_{q} U(N)} \cong F u n\left(S L_{q}(N)\right) \text {, }
$$

where the last but one object is the dense unital *-subalgebra of $C\left(S_{q} U(N)\right)$, generated by all $u_{i j}$.

2. Therefore the $*$-Hopf algebra structure on $A\left(q \in \mathbb{R} \backslash\{0\}, q>0\right.$ for $\left.B_{n}\right)$ is the same as in [RTF] (for $A_{n}, \varepsilon_{1}=\varepsilon_{2}=\cdots=\varepsilon_{N}=1$, also the same as in [W3]). It is called $\operatorname{Fun}\left(S U_{q}\left(N ; \varepsilon_{1}, \ldots, \varepsilon_{N}\right)\right)$ for $A_{n}, F u n\left(O_{q}\left(N ; \varepsilon_{1}, \ldots, \varepsilon_{N}\right)\right)$ for $B_{n}$ and $D_{n}, F u n\left(S p_{q}\left(n ; \varepsilon_{1}, \ldots, \varepsilon_{2 n}\right)\right)$ for $C_{n}$ (in [RTF] the symbol $S O_{q}$ instead of $O_{q}$ is used; Fun $\left(\operatorname{Sp} p_{q}\left(n ; \varepsilon_{1}, \ldots, \varepsilon_{2 n}\right)\right)$ are not explicitly defined there). Let us notice that, according to Thm. 1.4.4, for $\varepsilon_{1}=\cdots=\varepsilon_{N}=1$ each of these $*$-Hopf algebras can be completed in maximal $C^{*}$-seminorm giving a compact matrix quantum group $G=(\bar{A}, u)$, where $\bar{A}$ is the unital $C^{*}$-algebra generated by $u_{i j}$ satisfying (2.1)-(2.3), (2.27) (then we have $\bar{A}=C\left(S_{q} U(N)\right)$; see [W3]) or (2.7)(2.9), (2.27) (then $\bar{A}=C\left(O_{q}(N)\right)$ or $\bar{A}=C\left(S p_{q}(n)\right)$ ). Similar compact matrix quantum groups (for $B_{n}, C_{n}, D_{n}$ ) were described in [Ro].

Remark 7. Let $Y$ be the set of Young diagrams consisting of at most $N$ rows (we identify Young diagrams which can be obtained from one another by adding or subtracting a number of full columns consisting of $N$ boxes). Then due to Thm. 1.5 of [W3], there exist $u^{\alpha}, \alpha \in Y$, such that $\left\{u^{\alpha}\right\}_{\alpha \in Y}$ is the set of all nonequivalent irreducible corepresentations of $\mathrm{A}=F u n\left(S U_{q}\left(N ; \varepsilon_{1}, \ldots, \varepsilon_{N}\right)\right)$, 
$q \in(0,1]$ (A as a Hopf algebra doesn't depend on $\varepsilon_{1}, \ldots, \varepsilon_{N}$ ). We have $u^{\square}=u$. Moreover, $\operatorname{dim} u^{\alpha}$ and multiplicities of $u^{\alpha}$ in $u^{\alpha^{\prime}}(1) u^{\alpha^{\prime \prime}}, \alpha, \alpha^{\prime}, \alpha^{\prime \prime} \in Y$, are the same as for $S U(N)$.

Remark 8. By virtue of $[\mathrm{RTF}],(2.6)$ and (2.13) we obtain $R^{-1}=$ $s_{H H} R\left(q^{-1}\right) s_{H H}$, where $R\left(q^{-1}\right)$ is given by (2.5) or (2.11) with $q$ replaced by $q^{-1}$. Therefore using $B \in \operatorname{Mor}\left(u^{c}, \bar{u}\right),(1.7)$ and (1.19) we get

$$
\left(R^{\bar{u} u}\right)_{i j, k l}=\varepsilon_{k} \varepsilon_{j}\left(R^{u^{c} u}\right)_{i j, k l}=\varepsilon_{k} \varepsilon_{j} R_{k i, l j}^{-1}=\varepsilon_{k} \varepsilon_{j} R\left(q^{-1}\right)_{i k, j l} \text {. }
$$

This formula will be used in Section 3.

\section{§3. Real Complexifications of Quantum Groups}

In this Section we describe a procedure which, when applied to any CQT *-Hopf algebra $(\mathbb{A}, \mathscr{R})$, produces an object $(\mathbb{B}, \mathscr{S})$ of the same kind. If $A$ is commutative and $\mathscr{R}=1$, this procedure can be decomposed into complexification and then realification. Thus we call $(\mathbb{B}, \mathscr{S})$ the real complexification of $(\mathbb{A}, \mathscr{R})$. Its properties are described by Theorems 3.1 and 3.3, which are the main result of the present paper. In the case of $(\mathbb{A}, \mathscr{R})$ from Examples 2.1$2.2, \mathrm{~B}$ corresponds to quantum groups $S L_{q}\left(N, \mathbb{C} ; \varepsilon_{1}, \ldots, \varepsilon_{N}\right), O_{q}\left(N, \mathbb{C} ; \varepsilon_{1}, \ldots, \varepsilon_{N}\right)$, $S p_{q}\left(n, \mathbb{C} ; \varepsilon_{1}, \ldots, \varepsilon_{2 n}\right)$. For $q=1$, the dependence on $\varepsilon_{1}, \ldots, \varepsilon_{N}$ disappears and we get relations, which are fulfilled for the classical groups $S L(N, \mathbb{C}), O(N, \mathbb{C})$, $\operatorname{Sp}(n, \mathbb{C})$.

Let $\mathbb{A}=(A, *, \phi, \kappa, e)$ be a $*$-Hopf algebra. Omitting $*$-structure we obtain a Hopf algebra $\mathbb{H}=\mathbb{A}^{\mathbb{C}}=(H, \phi, \kappa, e)$ called the complexification of $\mathbb{A}$ (as algebras $H=A$ ). According to the Appendix we have moreover the conjugate Hopf algebra $\mathbb{H}^{j}=\left(H^{j}, \phi^{j}, \kappa^{j}, e^{j}\right)\left(\kappa^{-1}=* \kappa *\right.$ exists $)$. Put $W=H \otimes H^{j}$. As we will see (Thm. 3.1 for $\mathscr{R}=1$ )

$$
\mathbb{W}=\left(W,\left(j^{-1} \otimes j\right) s,(i d \otimes s \otimes i d)\left(\phi \otimes \phi^{j}\right), \kappa \otimes \kappa^{j}, e \otimes e^{j}\right)
$$

(cf. (0.2)) is a $*$-Hopf algebra. If $A$ is commutative then there exists a unique homomorphism of $*$-Hopf algebras $\tau: W \rightarrow A$ such that $\tau(x \otimes I)=x, x \in A=\mathbb{H}$. Thus $\tau$ defines a canonical 'embedding' of the quantum group corresponding to $\mathbb{A}$ into the quantum group of $\mathbb{W}$. We call $\mathbb{W}$ the realification of $H$ and write $\mathbb{W}=\mathbb{H}^{\mathbb{R}}$ (W doesn't depend on the $*$-structure of $\left.\mathbb{A}\right)$. But for a noncommutative $A$, such an 'embedding' doesn't exist (otherwise, for $x, y \in A$, $\left.x^{*}=\tau\left((x \otimes I)^{*}\right)=\tau\left(I \otimes x^{j}\right), x^{*} y=\tau\left(I \otimes x^{j}\right) \tau(y \otimes I)=\tau(y \otimes I) \tau\left(I \otimes x^{j}\right)=y x^{*}\right)$. Nevertheless, if $\mathbb{A}$ has CQT $*$-Hopf algebra structure, then using it we can 'twist' the definition of $\mathbb{W}$ in such a way that the obtained quantum group will contain the quantum group of $\mathbb{A}$. It occurs that this construction depends not only on $\mathbf{H}$ but also on the $*$-structure of $\mathbf{A}$. Thus we have a procedure, which for commutative $\mathbb{A}$ (and $\mathscr{R}=1$ ) can be decomposed into the complexification and then the realification. It is called the real complexification and is described as follows. 


\section{Theorem 3.1.}

1) Assume that $(\mathbf{A}, \mathscr{R})$ is a *-Hopf algebra with compatible hermitian element. Let $\mathbf{A}=(A, *, \phi, \kappa, e), I=I_{A}, H=A, \mathbf{H}=(H, \phi, \kappa, e), \mathbf{H}^{j}=\left(H^{j}, \phi^{j}, \kappa^{j}, e^{j}\right)$ be the Hopf algebra conjugate to the Hopf algebra $\mathbf{H}, j: H \rightarrow H^{j}$ be the corresponding antilinear bijection,

$$
\begin{gathered}
i=i d: H \rightarrow A, \\
i^{\prime}=* \circ i \circ j^{-1}: H^{j} \rightarrow A
\end{gathered}
$$

(these are canonical isomorphisms of Hopf algebras),

$$
\begin{gathered}
\phi_{2}=\left(i d \otimes s_{A A} \otimes i d\right)(\phi \otimes \phi): A \otimes A=A_{2} \rightarrow A_{2} \otimes A_{2}, \\
C=s_{A A}\left(\mathscr{R}^{-1} \otimes i d_{A_{2}} \otimes \mathscr{R}\right)\left(i d_{A_{2}} \otimes \phi_{2}\right) \phi_{2}: A_{2} \rightarrow A_{2}, \\
\rho=\left(i^{-1} \otimes i^{\prime-1}\right) C\left(i^{\prime} \otimes i\right): H^{j} \otimes H \rightarrow H \otimes H^{j}, \\
B=H \otimes H^{j} \text { as a vector space, } \\
m_{B}=\left(m_{H} \otimes m_{H^{j}}\right)\left(i d_{H} \otimes \rho \otimes i d_{H^{j}}\right): B \otimes B \rightarrow B, \\
*_{B}=\left(j^{-1} \otimes j\right) s_{H H^{j}}: B \rightarrow B, \\
\Phi=\left(i d_{H} \otimes s_{H H^{j}} \otimes i d_{H^{j}}\right)\left(\phi \otimes \phi^{j}\right): B \rightarrow B \otimes B, \\
E=e \otimes e^{j}: B \rightarrow \mathbb{C}, \\
K=\rho\left(\kappa^{j} \otimes \kappa\right) s_{H H^{j}}: B \rightarrow B .
\end{gathered}
$$

Then $m_{B}$ endows $B$ with the structure of an algebra with unity

$$
I_{B}=I \otimes I^{j}
$$

Moreover, $\mathbf{B}=\left(B, *_{B}, \Phi, E, K\right)$ is a $*$-Hopf algebra.

We define a homomorphism of Hopf algebras $\psi: A \rightarrow B$ by

$$
\psi(i(x))=x \otimes I^{j}, \quad x \in H .
$$

Let $\left\{u^{\alpha}\right\}_{\alpha \in J}$ be the set of all corepresentations of A. We put $u^{\alpha \cdot \beta}=u^{\alpha}(1) u^{\beta}$, $\alpha, \beta \in J$. Set

$$
w_{m n}^{\alpha}=\psi\left(u_{m n}^{\alpha}\right), \quad m, n=1, \ldots, \operatorname{dim} u^{\alpha}, \alpha \in J .
$$

Then $w^{\alpha}, \alpha \in J$, are corepresentations of B. Moreover

$$
\begin{gathered}
S w^{\alpha}=w^{\beta} S \quad \text { if } \quad S \in \operatorname{Mor}\left(u^{\alpha}, u^{\beta}\right), \\
w^{\alpha \cdot \beta}=w^{\alpha} \bigoplus w^{\beta}, \\
R^{\bar{\beta} \alpha}\left(\overline{w^{\beta}} \bigoplus w^{\alpha}\right)=\left(w^{\alpha} \bigoplus \overline{w^{\beta}}\right) R^{\bar{\beta} \alpha},
\end{gathered}
$$

where $\alpha, \beta \in J$ and $\left\{R^{\alpha \beta}\right\}_{\alpha, \beta \in J}$ is the compatible hermitian family corresponding to $\mathscr{R}$ (we sometimes identify $u^{\alpha}$ with $\alpha$ ). 
Denote $w^{\alpha, \beta}=w^{\alpha} \oplus \overline{w^{\beta}}$. Then

$$
\begin{gathered}
\overline{w^{\alpha, \beta}}=s_{\alpha \beta}^{-1}\left(w^{\beta, \alpha}\right) s_{\alpha \beta}, \\
w^{\alpha, \beta}(1) w^{\gamma, \delta}=D_{\alpha \beta \gamma \delta}^{-1}\left(w^{\alpha \cdot \gamma, \delta \cdot \beta}\right) D_{\alpha \beta \gamma \delta},
\end{gathered}
$$

where $D_{\alpha \beta \gamma \delta}=\left(i d_{\alpha} \otimes i d_{\gamma} \otimes s_{\beta \delta}\right)\left(i d_{\alpha} \otimes R^{\bar{\beta} \gamma} \otimes i d_{\delta}\right)$.

Let $\left\{u^{\alpha}\right\}_{\alpha \in I}$ be the set of all nonequivalent irreducible corepresentations of A. Then

$$
w^{\alpha, \beta}, \quad \alpha, \beta \in I,
$$

are nonequivalent, irreducible corepresentations of $B$

2) Moreover, if $\mathbb{A}$ is $C R$, then $\mathbb{B}$ is also $C R$ and (3.19) gives all nonequivalent, irreducible corepresentations of $\mathbb{B}$. Let

$$
u^{\alpha} \text { (1) } u^{\beta} \simeq \bigoplus_{\rho \in I} m_{\alpha \beta}^{\rho} u^{\rho}, \quad m_{\alpha \beta}^{\rho} \in \mathbb{N}, \quad \alpha, \beta \in I .
$$

Then

$$
w^{\alpha, \beta} \bigoplus w^{\gamma, \delta} \simeq \bigoplus_{\rho, \tau \in I} m_{\alpha \gamma}^{\rho} m_{\delta \beta}^{\tau} w^{\rho, \tau}
$$

$\alpha, \beta, \gamma, \delta \in I$

Corollary 3.2. With the assumptions of Thm. 3.1.1

1) $B$ can be identified with the universal *-algebra generated by the symbols $a^{\prime}(a \in A)$ satisfying $I^{\prime}=I$,

$$
\begin{aligned}
a^{\prime}+b^{\prime} & =(a+b)^{\prime}, \\
\lambda \cdot a^{\prime} & =(\lambda \cdot a)^{\prime}, \\
a^{\prime} \cdot b^{\prime} & =(a \cdot b)^{\prime}, \\
R^{\bar{\beta} \alpha}\left(\overline{\left(u^{\beta}\right)^{\prime}} \text { (1) }\left(u^{\alpha}\right)^{\prime}\right) & =\left(\left(u^{\alpha}\right)^{\prime} \oplus \overline{\left(u^{\beta}\right)^{\prime}}\right) R^{\bar{\beta} \alpha,}
\end{aligned}
$$

where $\lambda \in \mathbb{C}, a, b \in A, \alpha, \beta \in J$,

$$
\left(u^{\alpha}\right)_{i j}^{\prime}=\left(u_{i j}^{\alpha}\right)^{\prime}, \quad i, j=1, \ldots, \operatorname{dim} u^{\alpha}
$$

and the identification is given by

$$
a^{\prime}=\psi(a), \quad a \in A .
$$

2) Let $\left(u^{\alpha_{0}}\right)_{i j}, i, j=1, \ldots, \operatorname{dim} u^{\alpha_{0}}$, generate $A$ as an algebra (for some $\left.\alpha_{0} \in J\right)$. Set $u=u^{\alpha_{0}}$. Then $B$ can be identified with the universal $*$-algebra generated by the symbols $a^{\prime}(a \in A)$ satisfying (3.22)-(3.24), $I^{\prime}=I$ and

$$
R^{u u}\left(\overline{(\bar{u})^{\prime}} \oplus u^{\prime}\right)=\left(u^{\prime} \oplus \overline{(\bar{u})^{\prime}}\right) R^{u u},
$$

where the identification is given by (3.27) and $u^{\prime}, \bar{u}^{\prime}$ are defined by (3.26). 
3) Let $N, P \in \mathbb{N} \backslash\{0\}, H=\mathbb{C}^{N}, s_{m}, r_{m} \in \mathbb{N}, C_{m} \in \operatorname{Lin}\left(H^{\otimes s_{m}}, H^{\otimes r_{m}}\right), m=1, \ldots, P$, and $A$ be the universal algebra with unity generated by $u_{i j}, i, j=1, \ldots, N$, satisfying

$$
C_{m} u^{\oplus s_{m}}=u^{\oplus r_{m}} C_{m}, \quad m=1, \ldots, P .
$$

Assume that $u$ is a corepresentation of A. Then $B$ can be identified with the universal *-algebra with unity $I$, generated by $w_{i j}, i, j=1, \ldots, N$, satisfying

$$
\begin{gathered}
C_{m} w^{\oplus s_{m}}=w^{\oplus r_{m}} C_{m}, \quad m=1, \ldots, P, \\
R^{\bar{u} u}(\bar{w} \oplus w)=(w \bigoplus \bar{w}) R^{\bar{u} u},
\end{gathered}
$$

with identification $w_{i j}=\psi\left(u_{i j}\right), i, j=1, \ldots, N$.

4) Suppose that $\mathbf{A}$ is $C R$. Then $B$ is the universal *-algebra generated by $w_{i j}^{\alpha}, i, j=1, \ldots, \operatorname{dim} u^{\alpha}, \alpha \in J$, satisfying (3.14)-(3.16) and $w^{0}=\left(I_{B}\right)$ (where $\left.u^{0}=\left(I_{A}\right)\right)$.

Theorem 3.3. Let $(\mathbb{A}, \mathscr{R})$ be CQT *-Hopf algebra. Assume the notation of Thm. 3.1.1. Then

$$
R^{\alpha \beta} \in \operatorname{Mor}\left(w^{\alpha} \oplus w^{\beta}, w^{\beta} \oplus w^{\alpha}\right), \quad \alpha, \beta \in J
$$

There exists a unique homomorphism of $*$-Hopf algebras $\tau: B \rightarrow A$ such that $\tau \psi=i d_{A}$, i.e.

$$
\tau\left(x \otimes I^{j}\right)=i(x), \quad x \in H
$$

We define

$$
\mathscr{S}=\left[\left(\mathscr{R}^{-1}\right)_{41} \mathscr{R}_{1^{\prime} 3} \mathscr{R}_{24^{\prime}} \mathscr{R}_{2^{\prime} 3^{\prime}}\right] \phi_{4}\left(i \otimes i^{\prime} \otimes i \otimes i^{\prime}\right) \in(B \otimes B)^{\prime}
$$

(the linear functional in the square bracket has the value $\mathscr{R}^{-1}\left(x_{4} \otimes x_{1}\right) \mathscr{R}\left(x_{1}^{\prime} \otimes\right.$ $\left.x_{3}\right) \mathscr{R}\left(x_{2} \otimes x_{4}^{\prime}\right) \mathscr{R}\left(x_{2}^{\prime} \otimes x_{3}^{\prime}\right) \quad$ on $\left.\quad x_{1} \otimes x_{2} \otimes x_{3} \otimes x_{4} \otimes x_{1}^{\prime} \otimes x_{2}^{\prime} \otimes x_{3}^{\prime} \otimes x_{4}^{\prime} \in A^{\otimes 8}\right)$. Then

$$
\mathscr{S}(\psi \otimes \psi)=\mathscr{R}
$$

Furthermore $(\mathbf{B}, \mathscr{S})$ is a CQT *-Hopf algebra, denoted by $(\mathbb{A}, \mathscr{R})^{\mathbb{C}}(\mathbb{C R}$ is one symbol) and called the real complexification of (A, $\mathscr{R})$.

Remark 1. With the assumptions of Thm. 3.3 we have the following chain of CQT Hopf algebras

$$
(\mathrm{A}, \mathscr{R}) \stackrel{\psi}{\rightarrow}(\mathrm{A}, \mathscr{R})^{\mathbb{C} \mathbb{R}} \stackrel{\psi}{\rightarrow}(\mathbb{A}, \mathscr{R})^{\mathbb{C} \mathbb{R} \mathbb{R}} \stackrel{\psi}{\rightarrow} \cdots
$$

and the following chain of $*$-Hopf algebras

$$
(\mathbb{A}, \mathscr{R}) \stackrel{\tau}{\leftarrow}(\mathbf{A}, \mathscr{R})^{\mathbb{C} \mathbb{R}} \stackrel{\tau}{\leftarrow}(\mathbb{A}, \mathscr{R})^{\mathbb{C} \mathbb{R} \mathbb{C}} \stackrel{\tau}{\leftarrow} \cdots
$$


Remark 2. In his unpublished notes [Ko] T. H. Koornwinder considers any *-Hopf algebra $A$ with a unitary corepresentation $u \in M_{N}(A)$ such that $u_{i j}$ generate $A$ as algebra. Then he introduces $B$ in the same way as in Corollary 3.2.2 but instead of $R^{u u}$ he deals with a hermitian matrix $R \in M_{N^{2}}(A)$. Then he proves that $B$ is a $*$-Hopf algebra (with $\Phi\left(a^{\prime}\right)=\phi(a)^{\prime \otimes \prime}$, which corresponds to (3.8)) and (for $R \in \operatorname{Mor}(u \oplus u, u \oplus u)$ ) the existence of the homomorphism of *-Hopf algebras $\tau: B \rightarrow A$ such that $\tau\left(a^{\prime}\right)=a, a \in A$.

Remark 3. Let $u^{F}$ be a unitary corepresentation of a CQT $*$-Hopf algebra (A, $\mathscr{R})$. Put $T=w^{F}, \tilde{T}=\overline{w^{F}}, R=s_{F F}\left(R^{F F}\right)^{-1}$. Then relations (1.18) of [RTF] (provided in another context) hold in $B$ (note that $\tilde{R}=s_{F F}\left(R^{\bar{F} F}\right)^{-1}, \tilde{\widetilde{R}}=$ $\left.\overline{\left(R^{F F}\right)^{-1}} s_{F F}\right)$

In order to prove Thm. 3.1 we shall need

Lemma 3.4.

$$
C^{\otimes i d}(v \otimes w)=\left(R^{v w}\right)^{-1}(w \otimes v) R^{v w}
$$

$v, w \in \operatorname{Rep} \mathbf{A}$ (cf. (0.3), (0.7)),

$$
\begin{gathered}
C(m \otimes i d)=(i d \otimes m)(C \otimes i d)(i d \otimes C), \\
C(i d \otimes m)=(m \otimes i d)(i d \otimes C)(C \otimes i d), \\
C(I \otimes x)=x \otimes I, \quad x \in A, \\
C(x \otimes I)=I \otimes x, \quad x \in A, \\
*_{2} C=C *_{2}, \\
e_{2} C=e_{2}, \\
\phi_{2} C=(C \otimes C) \phi_{2}
\end{gathered}
$$

( $m$ denotes multiplication in A).

Proof. We use Einstein's convention. Let $u, v, w \in \operatorname{Rep} \mathbf{A}$. One has

$$
\phi_{2}(v \otimes w)_{i j, k l}=(v \otimes w)_{i j, m n} \otimes(v \otimes w)_{m n, k l}
$$

$\left(i d \otimes \phi_{2}\right) \phi_{2}(v \otimes w)_{i j, k l}=(v \otimes w)_{i j, m n} \otimes(v \otimes w)_{m n, p q} \otimes(v \otimes w)_{p q, k l}$.

Using (0.7), (1.16)-(1.17), one gets (3.38). Setting $v=1(w=1$, resp.) in (3.38) and using (1.15) ((1.18), resp.), we get (3.41) ((3.42), resp.). Using (3.38), (1.10) and (3.38) twice, we obtain

$[C(m \otimes i d)]^{\otimes i d}(u \otimes v \otimes w)$

$$
\begin{aligned}
& =C^{\otimes i d}((u \oplus v) \otimes w) \\
& =\left(R^{u \oplus v, w}\right)^{-1}(w \otimes(u \oplus v)) R^{u \oplus v, w} \\
& =(i d \otimes m)^{\otimes i d}\left\{\left[i d \otimes\left(R^{v w}\right)^{-1}\right]\left[\left(R^{u w}\right)^{-1} \otimes i d\right](w \otimes u \otimes v)\left[R^{u w} \otimes i d\right]\left[i d \otimes R^{v w}\right]\right\} \\
& =[(i d \otimes m)(C \otimes i d)(i d \otimes C)]^{\otimes i d}(u \otimes v \otimes w)
\end{aligned}
$$


and (3.39) follows. Similarly, using (3.38), (1.11) and (3.38) twice, we get (3.40). Moreover, applying $\phi_{2}$ to (3.38) and using (3.46), (3.38), one has

$$
\begin{aligned}
\left(\phi_{2} C\right)(v \otimes w)_{i j, k l} & =\left(R^{v w}\right)_{i j, a b}^{-1}(w \otimes v)_{a b, m n} \otimes(w \otimes v)_{m n, r s}\left(R^{v w}\right)_{r s, k l} \\
& =C(v \otimes w)_{i j, p q} \otimes C(v \otimes w)_{p q, k l} \\
& =(C \otimes C) \phi_{2}(v \otimes w)_{i j, k l}
\end{aligned}
$$

and so (3.45) is satisfied. Furthermore

$$
\begin{aligned}
\left(e_{2} C\right)(v \otimes w)_{i j, k l} & =\left(R^{v w}\right)_{i j, m n}^{-1} e_{2}\left[(w \otimes v)_{m n, p q}\right]\left(R^{v w}\right)_{p q, k l} \\
& =\left(R^{v w}\right)_{i j, m n}^{-1} \delta_{m p} \delta_{n q}\left(R^{v w}\right)_{p q, k l} \\
& =\delta_{i k} \delta_{j l}=e_{2}\left[(v \otimes w)_{i j, k l}\right]
\end{aligned}
$$

and so (3.44) follows. In virtue of (3.38), (1.13) and (3.38)

$$
\begin{aligned}
C *_{2}(v \otimes w)_{i j, k l}=C(\bar{w} \otimes \bar{v})_{j i, l k} & =\left(R^{\bar{w} \bar{v}}\right)_{j i, n m}^{-1}(\bar{v} \otimes \bar{w})_{n m, q p}\left(R^{\bar{w} \bar{v}}\right)_{q p, l k} \\
& =*_{2}\left[\left(R^{v w}\right)_{i j, m n}^{-1}(w \otimes v)_{m n, p q}\left(R^{v w}\right)_{p q, k l}\right] \\
& =*_{2} C(v \otimes w)_{i j, k l}
\end{aligned}
$$

and so (3.43) holds.

Since $i, i^{\prime}$ are Hopf algebra isomorphisms, Lemma 3.4 yields

\section{Corollary 3.5.}

$$
\begin{gathered}
\rho^{\otimes i d}\left(v^{j} \otimes w\right)=\left(R^{\bar{v} w}\right)^{-1}\left(w \otimes v^{j}\right) R^{\bar{v} w}, \quad v, w \in R e p \mathbf{H}, \\
\rho\left(m_{H^{j}} \otimes i d_{H}\right)=\left(i d_{H} \otimes m_{H^{j}}\right)\left(\rho \otimes i d_{H^{j}}\right)\left(i d_{H^{j}} \otimes \rho\right), \\
\rho\left(i d_{H^{j}} \otimes m_{H}\right)=\left(m_{H} \otimes i d_{H^{j}}\right)\left(i d_{H} \otimes \rho\right)\left(\rho \otimes i d_{H}\right), \\
\rho\left(I^{j} \otimes x\right)=x \otimes I^{j}, \quad x \in H, \\
\rho\left(x^{j} \otimes I\right)=I \otimes x^{j}, \quad x \in H, \\
\left(j^{-1} \otimes j\right) s_{H H^{j}} \rho s_{H H^{j}}=\rho\left(j \otimes j^{-1}\right), \\
\left(e \otimes e^{j}\right) \rho=e^{j} \otimes e,
\end{gathered}
$$

$\left(i d_{H} \otimes s_{H H^{j}} \otimes i d_{H^{j}}\right)\left(\phi \otimes \phi^{j}\right) \rho=(\rho \otimes \rho)\left(i d_{H^{j}} \otimes s_{H^{j} H} \otimes i d_{H}\right)\left(\phi^{j} \otimes \phi\right)$.

Proof of Theorem 3.1. Denote $i d=i d_{H}, m=m_{H}, i d_{j}=i d_{H^{j}}, m_{j}=m_{H^{j}}$.

1) By virtue of (3.48), the associativity of $m, m_{j}$ and (3.49), we get

$m_{B}\left(m_{B} \otimes i d_{B}\right)$

$=\left(m \otimes m_{j}\right)\left(m \otimes \rho\left(m_{j} \otimes i d\right) \otimes i d_{j}\right)\left(i d \otimes \rho \otimes i d_{j} \otimes i d \otimes i d_{j}\right)$

$=\left(m \otimes m_{j}\right)\left(m \otimes i d \otimes m_{j} \otimes i d_{j}\right)\left(i d \otimes i d \otimes \rho \otimes i d_{j} \otimes i d_{j}\right)\left(i d \otimes \rho \otimes \rho \otimes i d_{j}\right)$ 


$$
\begin{aligned}
& =\left(m \otimes m_{j}\right)\left(i d \otimes\left(m \otimes i d_{j}\right)(i d \otimes \rho)(\rho \otimes i d) \otimes m_{j}\right)\left(i d \otimes i d_{j} \otimes i d \otimes \rho \otimes i d_{j}\right) \\
& =\left(m \otimes m_{j}\right)\left(i d \otimes \rho \otimes i d_{j}\right)\left(i d \otimes i d_{j} \otimes\left(m \otimes m_{j}\right)\left(i d \otimes \rho \otimes i d_{j}\right)\right) \\
& =m_{B}\left(i d_{B} \otimes m_{B}\right)
\end{aligned}
$$

and so $B$ is an algebra. Moreover, by virtue of (3.50)-(3.51)

$$
\begin{aligned}
& m_{B}\left(I \otimes I^{j} \otimes x \otimes y^{j}\right)=\left(m \otimes m_{j}\right)\left(I \otimes x \otimes I^{j} \otimes y^{j}\right)=x \otimes y^{j}, \\
& m_{B}\left(x \otimes y^{j} \otimes I \otimes I^{j}\right)=\left(m \otimes m_{j}\right)\left(x \otimes I \otimes y^{j} \otimes I^{j}\right)=x \otimes y^{j},
\end{aligned}
$$

hence (3.11) follows. Obviously $*_{B}$ is an antilinear involution such that

$$
*_{B}\left(x \otimes y^{j}\right)=y \otimes x^{j}, \quad x, y \in H
$$

Next, let $x, y, z, t \in H$ and let $a_{m}, b_{m}, m=1, \ldots, E$, be elements of $H$ such that

$$
\rho\left(y^{j} \otimes z\right)=\sum_{m} a_{m} \otimes b_{m}^{j} .
$$

Applying (3.52) to $z \otimes y^{j}$, we get

$$
\rho\left(z^{j} \otimes y\right)=\left(j^{-1} \otimes j\right) s_{H H^{J}} \rho\left(y^{j} \otimes z\right)=\sum_{m} b_{m} \otimes a_{m}^{j} .
$$

Therefore

$$
\begin{aligned}
& m_{B}\left(*_{B} \otimes *_{B}\right) s_{B B}\left(x \otimes y^{j} \otimes z \otimes t^{j}\right) \\
& =\left(m \otimes m_{j}\right)\left(i d \otimes \rho \otimes i d_{j}\right)\left(t \otimes z^{j} \otimes y \otimes x^{j}\right) \\
& =\left(m \otimes m_{j}\right) \sum_{m} t \otimes b_{m} \otimes a_{m}^{j} \otimes x^{j}=\sum_{m} t b_{m} \otimes\left(x a_{m}\right)^{j} \\
& \quad=*_{B} \sum_{m} x a_{m} \otimes b_{m}^{j} t^{j}=*_{B}\left(m \otimes m_{j}\right)\left[x \otimes \rho\left(y^{j} \otimes z\right) \otimes t^{j}\right] \\
& =*_{B} m_{B}\left(x \otimes y^{j} \otimes z \otimes t^{j}\right)
\end{aligned}
$$

and it follows that $\left(B, *_{B}\right)$ is a $*$-algebra with unity.

Now we shall investigate properties of linear mappings $\Phi, E, K$. Obviously $\Phi, E$ are unital. Let $v, w, z, t \in \operatorname{Rep} \mathrm{H}$. Notice that $v^{j}, w^{j}, z^{j}, t^{j} \in$ Rep $\mathbb{H}^{j}$. Therefore using (0.3), (3.8) and (0.3), we get

$$
\begin{gathered}
\Phi\left(v \otimes w^{j}\right)_{a b, c d}=\sum_{k, l}\left(v \otimes w^{j}\right)_{a b, k l} \otimes\left(v \otimes w^{j}\right)_{k l, c d}, \\
E\left(v \otimes w^{j}\right)_{a b, c d}=\delta_{a c} \delta_{b d}
\end{gathered}
$$

One easily checks that (A.1)-(A.2) hold on $\left(v \otimes w^{j}\right)_{a b, c d}$. Since elements of this form span B, (A.1)-(A.2) are satisfied. Moreover, using (3.56), (3.10), (3.49), (3.51), (3.57) and (3.11), 


$$
\begin{aligned}
m_{B}\left(K \otimes i d_{B}\right) \Phi\left(v_{a c} \otimes w_{b d}^{j}\right)= & \sum_{k, l} m_{B}\left(K \otimes i d_{B}\right)\left(v_{a k} \otimes w_{b l}^{j} \otimes v_{k c} \otimes w_{l d}^{j}\right) \\
= & \sum_{k, l}\left(i d \otimes m_{j}\right)\left[\left(m \otimes i d_{j}\right)(i d \otimes \rho)(\rho \otimes i d) \otimes i d_{j}\right] \\
& \times\left[\left(w^{j}\right)_{b l}^{-1} \otimes\left(v^{-1}\right)_{a k} \otimes v_{k c} \otimes w_{l d}^{j}\right] \\
= & \sum_{l}\left(i d \otimes m_{j}\right)\left(\rho \otimes i d_{j}\right)\left(\left(w^{j}\right)_{b l}^{-1} \otimes \delta_{a c} I \otimes w_{l d}^{j}\right) \\
= & \sum_{l}\left(i d \otimes m_{j}\right)\left(\delta_{a c} I \otimes\left(w^{j}\right)_{b l}^{-1} \otimes w_{l d}^{j}\right) \\
= & \delta_{a c} I \otimes \delta_{b d} I^{j}=E\left(v_{a c} \otimes w_{b d}^{j}\right) I_{B}
\end{aligned}
$$

and hence $m_{B}\left(K \otimes i d_{B}\right) \Phi(x)=E(x) I_{B}$ for all $x \in B$. Similarly, using (3.56), (3.10), (3.48), (3.50), (3.57) and (3.11), one gets $m_{B}\left(i d_{B} \otimes K\right) \Phi(x)=E(x) I_{B}, x \in B$. Due to (3.50)-(3.51) and (3.6),

$$
\begin{aligned}
\left(x \otimes I^{j}\right)(I \otimes y) & =(x \otimes y), \\
\left(x \otimes I^{j}\right)\left(x^{\prime} \otimes I^{j}\right) & =\left(x x^{\prime} \otimes I^{j}\right), \\
(I \otimes y)\left(I \otimes y^{\prime}\right) & =\left(I \otimes y y^{\prime}\right), \\
(I \otimes y)\left(x \otimes I^{j}\right) & =\rho(y \otimes x),
\end{aligned}
$$

$x, x^{\prime} \in H, y, y^{\prime} \in H^{j}$. We have also

$$
\begin{gathered}
\Phi\left(x \otimes I^{j}\right) \Phi(I \otimes y)=\Phi(x \otimes y), \\
\Phi\left(x \otimes I^{j}\right) \Phi\left(x^{\prime} \otimes I^{j}\right)=\Phi\left(x x^{\prime} \otimes I^{j}\right), \\
\Phi(I \otimes y) \Phi\left(I \otimes y^{\prime}\right)=\Phi\left(I \otimes y y^{\prime}\right), \\
\Phi(I \otimes y) \Phi\left(x \otimes I^{j}\right)=\Phi[\rho(y \otimes x)] .
\end{gathered}
$$

Namely, if $\phi(x)=\sum_{m} a_{m} \otimes b_{m}, \phi^{j}(y)=\sum_{n} c_{n} \otimes d_{n}$, then using (3.8), (3.58) and (3.8), one gets (3.62). Similarly, by virtue of (3.8), (3.59) (or (3.60)), multiplicativity of $\phi\left(\right.$ or $\phi^{j}$ ) and (3.8), one checks (3.63)-(3.64). Furthermore, using (3.8), (3.61), (3.54) and (3.8),

$$
\begin{aligned}
\Phi(I \otimes y) \Phi\left(x \otimes I^{j}\right) & =\left[\sum_{n}\left(I \otimes c_{n}\right) \otimes\left(I \otimes d_{n}\right)\right]\left[\sum_{m}\left(a_{m} \otimes I^{j}\right) \otimes\left(b_{m} \otimes I^{j}\right)\right] \\
& =\sum_{m, n} \rho\left(c_{n} \otimes a_{m}\right) \otimes \rho\left(d_{n} \otimes b_{m}\right) \\
& =(\rho \otimes \rho)(i d \otimes s \otimes i d)\left(\phi^{j} \otimes \phi\right)(y \otimes x) \\
& =\Phi[\rho(y \otimes x)]
\end{aligned}
$$


so (3.65) is fulfilled. Let

$$
\rho\left(y \otimes x^{\prime}\right)=\sum_{p} f_{p} \otimes g_{p} .
$$

By virtue of (3.62), (3.65), (3.66), (3.62), (3.63)-(3.64) and (3.62),

$$
\begin{aligned}
\Phi(x \otimes y) \Phi\left(x^{\prime} \otimes y^{\prime}\right) & =\Phi\left(x \otimes I^{j}\right) \Phi(I \otimes y) \Phi\left(x^{\prime} \otimes I^{j}\right) \Phi\left(I \otimes y^{\prime}\right) \\
& =\sum_{p} \Phi\left(x \otimes I^{j}\right) \Phi\left(f_{p} \otimes g_{p}\right) \Phi\left(I \otimes y^{\prime}\right) \\
& =\sum_{p} \Phi\left(x \otimes I^{j}\right) \Phi\left(f_{p} \otimes I^{j}\right) \Phi\left(I \otimes g_{p}\right) \Phi\left(I \otimes y^{\prime}\right) \\
& =\sum_{p} \Phi\left(x f_{p} \otimes g_{p} y^{\prime}\right) .
\end{aligned}
$$

Similarly, using (3.58), (3.61), (3.66), (3.58), (3.59)-(3.60) and (3.58), one has

$$
(x \otimes y)\left(x^{\prime} \otimes y^{\prime}\right)=\sum_{p} x f_{p} \otimes g_{p} y^{\prime} .
$$

Therefore $\Phi$ is a homomorphism. Moreover, in virtue of (3.67), (3.9), (3.66), (3.53) and (3.9),

$$
\begin{aligned}
E\left[(x \otimes y)\left(x^{\prime} \otimes y^{\prime}\right)\right] & =\sum_{p} E\left(x f_{p} \otimes g_{p} y^{\prime}\right) \\
& =\sum_{p} e\left(x f_{p}\right) e^{j}\left(g_{p} y^{\prime}\right) \\
& =e(x)\left[\left(e \otimes e^{j}\right) \rho\left(y \otimes x^{\prime}\right)\right] e^{j}\left(y^{\prime}\right) \\
& =e(x)\left[\left(e^{j} \otimes e\right)\left(y \otimes x^{\prime}\right)\right] e^{j}\left(y^{\prime}\right) \\
& =E(x \otimes y) E\left(x^{\prime} \otimes y^{\prime}\right) .
\end{aligned}
$$

Let $v, w \in \operatorname{Rep} \mathbf{H}$. Due to (3.55), (3.56), (3.55) and (3.56),

$$
\begin{aligned}
\Phi *_{B}\left(v \otimes w^{j}\right)_{a b, c d} & =\Phi\left(w \otimes v^{j}\right)_{b a, d c}=\sum_{k, l}\left(w \otimes v^{j}\right)_{b a, l k} \otimes\left(w \otimes v^{j}\right)_{l k, d c} \\
& =\left(*_{B} \otimes *_{B}\right) \sum_{k, l}\left(v \otimes w^{j}\right)_{a b, k l} \otimes\left(v \otimes w^{j}\right)_{k l, c d} \\
& =\left(*_{B} \otimes *_{B}\right) \Phi\left(v \otimes w^{j}\right)_{a b, c d},
\end{aligned}
$$

and hence $\mathbf{B}$ is a $*$-Hopf algebra.

According to (3.59), (3.8) and (3.9), the mapping $H \ni x \rightarrow x \otimes I^{j} \in B$ is a homomorphism of Hopf algebras. Hence $\psi: A \rightarrow B$ is also a homomorphism of Hopf algebras (but due to (3.55), $\psi_{*} \neq *_{B} \psi$ for $A \neq \mathbb{C} I$ ). Consequently, $u^{\alpha} \in \operatorname{Rep} \mathbf{A}(\alpha \in J)$ yields $w^{\alpha} \in \operatorname{Rep} \mathbf{B}$. Moreover,

$$
\left(w^{\alpha} \bigoplus \overline{w^{\beta}}\right)_{k l, m n}=\psi\left(u_{k m}^{\alpha}\right) \psi\left(u_{l n}^{\beta}\right)^{*}=\left(u_{k m}^{\alpha} \otimes I^{j}\right)\left(I \otimes\left(u_{l n}^{\beta}\right)^{j}\right)=u_{k m}^{\alpha} \otimes\left(u_{l n}^{\beta}\right)^{j},
$$


and so

$$
w^{\alpha} \oplus \overline{w^{\beta}}=u^{\alpha} \otimes\left(u^{\beta}\right)^{j}
$$

Let $S \in \operatorname{Mor}\left(u^{\alpha}, u^{\beta}\right), \alpha, \beta \in J$. Applying $\psi^{\otimes i d}$ to the relations $S u^{\alpha}=u^{\beta} S, u^{\alpha \cdot \beta}=$ $u^{\alpha}\left(\mathbb{D} u^{\beta}\right.$, we obtain (3.14)-(3.15). Next, by virtue of (3.61), (3.47) and (3.68),

$$
\begin{aligned}
\left(\overline{w^{\beta}} \oplus w^{\alpha}\right)_{k l, m n} & =\psi\left(u_{k m}^{\beta}\right)^{*} \psi\left(u_{l n}^{\alpha}\right)=\left(I \otimes\left(u_{k m}^{\beta}\right)^{j}\right)\left(u_{l n}^{\alpha} \otimes I^{j}\right) \\
& =\rho\left(\left(u_{k m}^{\beta}\right)^{j} \otimes u_{l n}^{\alpha}\right)=\sum_{a, b, c, d}\left(R^{\bar{\beta} \alpha}\right)_{k l, a b}^{-1}\left[u_{a c}^{\alpha} \otimes\left(u_{b d}^{\beta}\right)^{j}\right] R_{c d, m n}^{\bar{\beta} \alpha} \\
& =\sum_{a, b, c, d}\left(R^{\bar{\beta} \alpha}\right)_{k l, a b}^{-1}\left(w^{\alpha} \bigoplus \overline{w^{\beta}}\right)_{a b, c d} R_{c d, m n}^{\bar{\beta} \alpha},
\end{aligned}
$$

and so (3.16) follows. Eq. (3.17) is a consequence of (0.6). According to (3.16), $\left(i d_{\alpha} \otimes R^{\bar{\beta} \gamma} \otimes i d_{\delta}\right)\left(w^{\alpha} \oplus \overline{w^{\beta}}\right.$ (1) $\left.w^{\gamma} \oplus \overline{w^{\delta}}\right)=\left(w^{\alpha} \oplus w^{\gamma}\right.$ (1) $\overline{w^{\beta}}$ (1) $\left.\overline{w^{\delta}}\right)\left(i d_{\alpha} \otimes R^{\bar{\beta} \gamma} \otimes i d_{\delta}\right)$, while due to $(0.6)$,

$$
\left(i d_{\alpha} \otimes i d_{\gamma} \otimes s_{\beta \delta}\right)\left(w^{\alpha} \text { (1) } w^{\gamma}\left(\overline{w^{\beta}} \text { (1) } \overline{w^{\delta}}\right)=\left(w^{\alpha} \oplus w^{\gamma} \text { (1) } \overline{w^{\delta}(1) w^{\beta}}\right)\left(i d_{\alpha} \otimes i d_{\gamma} \otimes s_{\beta \delta}\right)\right. \text {. }
$$

Therefore (3.18) holds.

Since the elements (A.6) are linearly independent, then (see (3.68))

$$
\left(w^{\alpha} \oplus \overline{w^{\beta}}\right)_{k l, m n}, \quad k, m=1, \ldots, \operatorname{dim} u^{\alpha} ; l, n=1, \ldots, \operatorname{dim} u^{\beta}, \quad \alpha, \beta \in I,
$$

are also linearly independent. Therefore the last statement of Thm. 3.1.1 is true.

2) If $\mathbf{A}$ is $\mathbf{C R}$, then the elements (A.6) form a basis in $A$. Thus using (3.68), matrix elements of (3.19) give a basis in B. Using Prop. A.2, we get the first statement of Thm. 3.1.2.

Assume (3.20). Then by (3.13),

$$
w^{\alpha}(1) w^{\beta} \simeq \bigoplus_{\rho \in I} m_{\alpha \beta}^{\rho} w^{\rho}, \quad \alpha, \beta \in I \text {. }
$$

Combining that with (3.18), we obtain

$$
w^{\alpha, \beta} \bigoplus w^{\gamma, \delta} \simeq w^{\alpha \cdot \gamma, \delta \cdot \beta}=w^{\alpha} \bigoplus w^{\gamma} \oplus \overline{w^{\delta} \bigoplus w^{\beta}} \simeq\left(\bigoplus_{\rho \in I} m_{\alpha \gamma}^{\rho} w^{\rho}\right) \bigoplus\left(\bigoplus_{\tau \in I} m_{\delta \beta}^{\tau} \overline{w^{\tau}}\right),
$$

$\alpha, \beta, \gamma, \delta \in I$, and (3.21) follows.

Proof of Corollary 3.2. 1) Let $B^{\prime}$ be the universal *-algebra under consideration. Since $\psi$ is a homomorphism and (3.16) holds, then $\psi(a), a \in A$, satisfy relations (3.22)-(3.25). Therefore there exists a $*$-homomorphism $\theta$ : $B^{\prime} \rightarrow B$ such that $\theta\left(a^{\prime}\right)=\psi(a)$. Due to $(3.25)$

$$
\left(u_{i k}^{\beta}\right)^{\prime *}\left(u_{j l}^{\alpha}\right)^{\prime}=\left(R^{\bar{\beta} \alpha}\right)_{i j, a b}^{-1}\left(u_{a c}^{\alpha}\right)^{\prime}\left(u_{b d}^{\beta}\right)^{\prime *} R_{c d, k l}^{\bar{\beta} \alpha}
$$


and thus

each element of $B^{\prime}$ is a combination of $a_{1}^{\prime} a_{2}^{\prime} \ldots a_{m}^{\prime} b_{1}^{\prime *} \ldots b_{n}^{\prime *}$,

$a_{1}, \ldots, a_{m}, b_{1}, \ldots, b_{n} \in A, m, n \in \mathbb{N}$. Let $\left\{e_{k}\right\}_{k \in K}$ be a linear basis in $A$. Then by (3.69) and (3.22)-(3.24), elements $e_{k}^{\prime} e_{l}^{\prime *}, k, l \in K$, generate $B^{\prime}$. But $\theta\left(e_{k}^{\prime} e_{l}^{\prime *}\right)=$ $e_{k} \otimes\left(e_{l}\right)^{j}$ form a basis in $B$ and therefore $\theta$ is a $*$-isomorphism which gives the desired identification.

2) We define $B^{\prime}$ and $*$-homomorphism $\theta$ as in 1 ). Put $v_{i j}=u_{i j}{ }^{*}$ (they also generate $A$ as algebra). Then any element of $B^{\prime}$ is a combination of products of elements $u_{i j}^{\prime}$ and $v_{k l}^{\prime}, i, j, k, l=1, \ldots$, dim $u$. Using (3.28) we can assume that in each such product all $u_{i j}^{\prime}$ are to the left of all $v_{k l}^{\prime *}$. The rest of the proof is the same as in 1).

3) Let $B^{\prime}$ be the universal *-algebra under consideration and let $B_{0}$ be the subalgebra of $B^{\prime}$ generated by all $w_{i j}$ and $I$. According to (3.31), $B^{\prime}=B_{0} B_{0}^{*}$. Since (3.30) has the same form as (3.29), then there exists a unital homomorphism of algebras $l: A \rightarrow B_{0}$ such that $l\left(u_{i j}\right)=w_{i j}, i, j=1, \ldots, N$.

Applying $\psi$ to (3.29) and using (3.16) for $u^{\alpha}=u^{\beta}=u$, we get that the matrix $w^{\prime}=\psi\left(u_{i j}\right)_{i, j=1}^{N}$ satisfies relations (3.30)-(3.31). Therefore there exists a unital $*$-homomorphism $\theta: B^{\prime} \rightarrow B$ such that $\theta\left(w_{i j}\right)=\psi\left(u_{i j}\right), i, j=1, \ldots, N$. Thus

$$
\theta \circ l=\psi
$$

(it holds on $\left.u_{i j}\right)$. Hence, if $\left\{e_{k}\right\}_{k \in K}$ is a linear basis in $A$, then $\left\{l\left(e_{k}\right)\right\}_{k \in K}$ is a linear basis in $B_{0}\left(l(A)=B_{0}\right)$ and $l\left(e_{k}\right) l\left(e_{m}\right)^{*}, k, m \in K$, generate $\mathbb{B}^{\prime}$. But using (3.70),

$$
\theta\left(l\left(e_{k}\right) l\left(e_{m}\right)^{*}\right)=\psi\left(e_{k}\right) \psi\left(e_{m}\right)^{*}=e_{k} \otimes\left(e_{m}\right)^{j}
$$

form a basis in $B$. Therefore $\theta$ is a $*$-isomorphism, which identifies $w_{i j}$ with $\psi\left(u_{i j}\right), i, j=1, \ldots, N$.

4) Let $B^{\prime}$ be the universal $*$-algebra generated by $z_{i j}^{\alpha}, i, j=1, \ldots, \operatorname{dim} u^{\alpha}$, $\alpha \in J$, satisfying (3.14)-(3.16) and $z^{0}=(I)$. But (3.14)-(3.16) and $w^{0}=(I)$ hold in $B$, thus there exists a $*$-homomorphism $\theta: B^{\prime} \rightarrow B$ such that $\theta\left(z_{i j}^{\alpha}\right)=w_{i j}^{\alpha}, i$, $j=1, \ldots, \operatorname{dim} u^{\alpha}, \alpha \in J$. Let $x \in B^{\prime}$. Then $x$ is a combination of products of $z_{i j}^{\alpha}$ and $z_{k l}^{\beta *}, i, j=1, \ldots, \operatorname{dim} u^{\alpha}, k, l=1, \ldots, \operatorname{dim} u^{\beta}, \alpha, \beta \in J$. Due to (3.16) one can assume that all $z_{i j}^{\alpha}$ are on the left of all $z_{k l}^{\beta *}$. Next, according to (3.15), each product can be written as $z_{m n}^{\gamma}\left(z_{p q}^{\delta}\right)^{*}$ for some $\gamma, \delta \in J$. Decomposing $u^{\gamma}, u^{\delta}$ into irreducible corepresentations and using (3.14), we obtain corresponding decompositions of $z^{\gamma}, z^{\delta}$. Therefore we can assume that (in the expression for $x$ ) $\gamma, \delta \in I$. Hence $z_{m n}^{\gamma}\left(z_{p q}^{\delta}\right)^{*}, m, n=1, \ldots, \operatorname{dim} u^{\gamma}, p, q=1, \ldots, \operatorname{dim} u^{\delta}, \gamma$, $\delta \in I$, linearly generate $B^{\prime}$. But

$$
\theta\left(z_{m n}^{\gamma}\left(z_{p q}^{\delta}\right)^{*}\right)=w_{m n}^{\gamma}\left(w_{p q}^{\delta}\right)^{*}=w_{m p, n q}^{\gamma, \delta}
$$


which, due to Thm. 3.1.2 and Prop. A.2, form a linear basis in B. We conclude that $\theta$ is a $*$-isomorphism, so we can identify $B^{\prime}$ with $B$ and $z_{i j}^{\alpha}$ with $w_{i j}^{\alpha}$.

Proof of Theorem 3.3. Since $(\mathbf{A}, \mathscr{R})$ is a CQT *-Hopf algebra, then

$$
R^{\alpha \beta}\left(u^{\alpha} \oplus u^{\beta}\right)=\left(u^{\beta} \oplus u^{\alpha}\right) R^{\alpha \beta} \text {, }
$$

$\alpha, \beta \in J$. Applying $\psi^{\otimes i d}$, we get

$$
R^{\alpha \beta}\left(w^{\alpha} \oplus w^{\beta}\right)=\left(w^{\beta} \oplus w^{\alpha}\right) R^{\alpha \beta}
$$

and so (3.32) follows. Moreover, (3.71) implies that equations (3.22)-(3.25), with $a^{\prime}$ replaced by $a, a \in A$, are satisfied. Therefore, by Corollary 3.2.1 there exists a $*$-homomorphism $\tau: B \rightarrow A$ such that

$$
\tau(\psi(a))=a .
$$

Hence (3.33) holds and $\tau$ is unital. Using (3.73) for $a$ equal to $u_{i j}^{\alpha}, i, j=$ $1, \ldots, \operatorname{dim} u^{\alpha}, \alpha \in J$, we get $\tau\left(w_{i j}^{\alpha}\right)=u_{i j}^{\alpha}$. Consequently,

$$
(\tau \otimes \tau) \Phi w_{i j}^{\alpha}=\sum_{k}(\tau \otimes \tau)\left(w_{i k}^{\alpha} \otimes w_{k j}^{\alpha}\right)=\sum_{k} u_{i k}^{\alpha} \otimes u_{k j}^{\alpha}=\phi u_{i j}^{\alpha}=\phi \tau\left(w_{i j}^{\alpha}\right)
$$

Moreover, $e \tau\left(w_{i j}^{\alpha}\right)=e\left(u_{i j}^{\alpha}\right)=\delta_{i j}=E\left(w_{i j}^{\alpha}\right)$. Since $w_{i j}^{\alpha}, i, j=1, \ldots, \operatorname{dim} u^{\alpha}, \alpha \in J$, generate $B$ as $*$-algebra and $\tau, \Phi, \phi, E, e$ are $*$-homomorphisms, then $\tau$ is a homomorphism of $*$-Hopf algebras. Since $x \otimes I^{j}, x \in H$, generate the $*$-algebra $B$, then uniqueness of $\tau$ follows.

According to (3.1)-(3.2) and (3.68),

$$
\left(i \otimes i^{\prime}\right) w_{k l, m n}^{\alpha, \beta}=u_{k m}^{\alpha} \otimes{\overline{u^{\beta}}}_{l n}, \quad \alpha, \beta \in J .
$$

Therefore, for $\alpha, \beta, \gamma, \delta \in J$,

$$
\begin{aligned}
& \mathscr{S}\left(w_{k l, m n}^{\alpha, \beta} \otimes w_{p q, r s}^{\gamma, \delta}\right) \\
& =\sum_{a, b, c, d}\left[(\mathscr{R})_{41}^{-1} \mathscr{R}_{1^{\prime} 3} \mathscr{R}_{24^{\prime}}, \mathscr{R}_{2^{\prime} 3^{\prime}}\right] \\
& \quad \times\left(u_{k a}^{\alpha} \otimes{\overline{u^{\beta}}}_{l b} \otimes u_{p c}^{\gamma} \otimes{\overline{u^{\delta}}}_{q d} \otimes u_{a m}^{\alpha} \otimes{\overline{u^{\beta}}}_{b n} \otimes u_{c r}^{\gamma} \otimes \bar{u}_{d s}^{\delta}\right) \\
& =\sum_{a, b, c, d}\left(R^{\bar{\delta} \alpha}\right)_{q k, a d}^{-1} R_{p a, m c}^{\alpha \gamma} R_{d l, b s}^{\bar{\beta} \bar{\delta}} R_{c b, n r}^{\bar{\beta} \gamma},
\end{aligned}
$$

and so

$$
S^{\alpha, \beta ; \gamma, \delta}=\left(i d_{\gamma} \otimes\left(R^{\bar{\delta} \alpha}\right)^{-1} \otimes i d_{\beta}\right)\left(R^{\alpha \gamma} \otimes R^{\bar{\beta} \bar{\delta}}\right)\left(i d_{\alpha} \otimes R^{\bar{\beta} \gamma} \otimes i d_{\delta}\right),
$$

where $\left\{S^{v ; w}\right\}_{v, w \in R e p \text { B }}$ is the family corresponding to $\mathscr{S}$ by (1.16).

In particular $S^{\alpha, 0 ; \gamma, 0}=R^{\alpha \gamma}$ (where $u^{0}=(I)$ ). Thus by (3.13),

$\mathscr{S}(\psi \otimes \psi)\left(u_{i j}^{\alpha} \otimes u_{k l}^{\beta}\right)=\mathscr{S}\left(w_{i, j}^{\alpha, 0} \otimes w_{k, l}^{\beta, 0}\right)=S_{k i, j l}^{\alpha, 0 ; \beta, 0}=R_{k i, j l}^{\alpha \beta}=\mathscr{R}\left(u_{i j}^{\alpha} \otimes u_{k l}^{\beta}\right)$, $\alpha, \beta \in J$, and (3.35) holds. 
By (3.68), $L=\left\{w^{\alpha, \beta}\right\}_{\alpha, \beta \in J}$ is a sufficient set of corepresentations of $\mathbf{B}$. Thus in order to prove that $(\mathbf{B}, \mathscr{S})$ is CQT $*$-Hopf algebra, it suffices (see Prop. 1.3.1. b) d)) to check (1.15), (1.10)-(1.13) for $v, w, v^{\prime}, w^{\prime} \in L$. We will denote these equations (in case of $S^{v ; w}, v, w \in L$ ) by $(1.15)^{\prime},(1.10)^{\prime}-(1.13)^{\prime}$.

Putting $\alpha=\beta=0$ in (3.74) and using (1.15), (1.18), we obtain (1.15)'. Applying $*_{B}$ to (3.72) and using (0.6), we get $s \overline{R^{\alpha \beta}} s \in \operatorname{Mor}\left(\overline{w^{\beta}} \oplus \overline{w^{\alpha}}, \overline{w^{\alpha}} \bigoplus \overline{w^{\beta}}\right)$. Thus, by (1.13), we obtain

$$
R^{\bar{\beta} \bar{\alpha}} \in \operatorname{Mor}\left(\overline{w^{\beta}} \oplus \overline{w^{\alpha}}, \overline{w^{\alpha}} \oplus \overline{w^{\beta}}\right) .
$$

Combining this with (3.16) and (3.32), one has

$$
S^{\alpha, \beta ; \gamma, \delta} \in \operatorname{Mor}\left(w^{\alpha} \oplus \overline{w^{\beta}} \bigoplus w^{\gamma} \bigoplus \overline{w^{\delta}}, w^{\gamma} \bigoplus \overline{w^{\delta}} \bigoplus w^{\alpha} \oplus \overline{w^{\beta}}\right)
$$

and therefore (1.12)' holds.

According to (3.74),

$$
S^{\alpha \cdot \alpha^{\prime}, \beta^{\prime} \cdot \beta ; \gamma, \delta}=\left(i d_{\gamma} \otimes\left(R^{\bar{\delta}, \alpha \cdot \alpha^{\prime}}\right)^{-1} \otimes i d_{\beta^{\prime} \beta}\right)\left(R^{\alpha \alpha^{\prime}, \gamma} \otimes R^{\overline{\beta^{\prime} \beta}, \bar{\delta}}\right)\left(i d_{\alpha \alpha^{\prime}} \otimes R^{\overline{\beta^{\prime} \beta}, \gamma} \otimes i d_{\delta}\right) .
$$

Using (0.6), (1.7) and (1.10)-(1.11), we obtain

$$
\begin{aligned}
& S^{w^{\alpha}} \oplus w^{\alpha^{\prime}} \oplus \overline{w^{\beta}} \oplus \overline{w^{\beta^{\prime}}} ; w^{\gamma} \oplus \overline{w^{\delta}} \\
& \quad=\left(i d_{\gamma} \otimes\left(R^{\bar{\delta}, \alpha \cdot \alpha^{\prime}}\right)^{-1} \otimes i d_{\beta \beta^{\prime}}\right)\left(R^{\alpha \alpha^{\prime}, \gamma} \otimes R^{\bar{\beta} \bar{\beta}^{\prime}, \bar{\delta}}\right)\left(i d_{\alpha \alpha^{\prime}} \otimes R^{\bar{\beta} \overline{\beta^{\prime}}, \gamma} \otimes i d_{\delta}\right) \\
& \quad=\left(R^{\bar{\delta} \alpha}\right)^{-1}\left(R^{\bar{\delta} \alpha^{\prime}}\right)^{-1} R^{\alpha \gamma} R^{\alpha^{\prime} \gamma} R^{\bar{\beta} \bar{\delta}} R^{\overline{\beta^{\prime}} \bar{\delta}} R^{\bar{\beta} \gamma} R^{\overline{\beta^{\prime} \gamma}},
\end{aligned}
$$

where in the last step we omitted identity operators acting in various spaces. But due to (3.16),

$$
i d_{\alpha} \otimes R^{\bar{\beta} \alpha^{\prime}} \otimes i d_{\beta^{\prime}} \in \operatorname{Mor}\left(w^{\alpha} \oplus \overline{w^{\beta}} \oplus w^{\alpha^{\prime}} \oplus \overline{w^{\beta^{\prime}}}, w^{\alpha} \text { (1) } w^{\alpha^{\prime}} \text { (1) } \overline{w^{\beta}} \text { (1) } \overline{w^{\beta^{\prime}}}\right) \text {. }
$$

Using that, (1.7) and braid relations (see (1.14))

$$
R^{\alpha^{\prime} \gamma} R^{\bar{\beta} \gamma} R^{\bar{\beta} \alpha^{\prime}}=R^{\bar{\beta} \alpha^{\prime}} R^{\bar{\beta} \gamma} R^{\alpha^{\prime} \gamma}, \quad\left(R^{\bar{\delta} \alpha^{\prime}}\right)^{-1} R^{\bar{\beta} \bar{\delta}} R^{\bar{\beta} \alpha^{\prime}}=R^{\bar{\beta} \alpha^{\prime}} R^{\bar{\beta} \bar{\delta}}\left(R^{\bar{\delta} \alpha^{\prime}}\right)^{-1},
$$

we get

$$
\begin{aligned}
\left(i d_{\gamma}\right. & \left.\otimes i d_{\delta} \otimes i d_{\alpha} \otimes R^{\bar{\beta} \alpha^{\prime}} \otimes i d_{\beta^{\prime}}\right) S^{\left(w^{\alpha} \oplus \overline{w^{\beta}}\right) \oplus\left(w^{\alpha^{\prime}} \oplus \overline{\left.w^{\beta^{\prime}}\right)} ; w^{\gamma} \oplus \overline{w^{\delta}}\right.} \\
= & \left(R^{\bar{\delta} \alpha}\right)^{-1}\left(R^{\bar{\delta} \alpha^{\prime}}\right)^{-1} R^{\alpha \gamma} R^{\alpha^{\prime} \gamma} R^{\bar{\beta} \bar{\delta}} R^{\overline{\beta^{\prime}} \bar{\delta}} R^{\bar{\beta} \gamma} R^{\overline{\beta^{\prime} \gamma} \gamma} R^{\bar{\beta} \alpha^{\prime}} \\
& =\left(R^{\bar{\delta} \alpha}\right)^{-1} R^{\alpha \gamma}\left(R^{\bar{\delta} \alpha^{\prime}}\right)^{-1} R^{\bar{\beta} \bar{\delta}} R^{\overline{\beta^{\prime} \delta}}\left[R^{\bar{\beta} \alpha^{\prime}} R^{\bar{\beta} \gamma} R^{\alpha^{\prime} \gamma}\right] R^{\overline{\beta^{\prime} \gamma} \gamma} \\
& =\left(R^{\bar{\delta} \alpha}\right)^{-1} R^{\alpha \gamma}\left[R^{\bar{\beta} \alpha^{\prime}} R^{\bar{\beta} \bar{\delta}}\left(R^{\bar{\delta} \alpha^{\prime}}\right)^{-1}\right] R^{\overline{\beta^{\prime}} \bar{\delta}} R^{\bar{\beta} \gamma} R^{\alpha^{\prime} \gamma} R^{\overline{\beta^{\prime} \gamma} \gamma} \\
& =\left(i d_{\gamma} \otimes i d_{\delta} \otimes i d_{\alpha} \otimes R^{\bar{\beta} \alpha^{\prime}} \otimes i d_{\beta^{\prime}}\right) S^{\alpha, \beta ; \gamma, \delta} S^{\alpha^{\prime}, \beta^{\prime} ; \gamma, \delta}
\end{aligned}
$$

and so $(1.10)^{\prime}$ holds. In a similar manner one proves $(1.11)^{\prime}$.

Let $\tilde{s}(x \otimes y \otimes z \otimes t)=t \otimes z \otimes y \otimes x . \quad$ By virtue of (1.7) for $s_{\gamma \delta} \in$ $\operatorname{Mor}\left(\overline{w^{\gamma, \delta}}, w^{\delta, \gamma}\right),(1.8)$ for $s_{\alpha \beta} \in \operatorname{Mor}\left(\overline{w^{\alpha, \beta}}, w^{\beta, \alpha}\right),(3.74),(1.13)$ and $(3.74)$, we get 


$$
\begin{aligned}
& s_{\alpha \beta, \gamma \delta} S^{\overline{w^{\gamma, \delta} ; \overline{w^{\alpha, \beta}}}} S_{\alpha \beta, \gamma \delta}=s_{\alpha \beta, \gamma \delta} s_{\alpha \beta}^{-1} s_{\gamma \delta}^{-1} S^{\delta, \gamma ; \beta, \alpha} s_{\alpha \beta} S_{\gamma \delta} s_{\alpha \beta, \gamma \delta} \\
& \quad=\tilde{s}\left(i d_{\beta} \otimes\left(R^{\bar{\alpha} \delta}\right)^{-1} \otimes i d_{\gamma}\right)\left(R^{\delta \beta} \otimes R^{\bar{\gamma} \bar{\alpha}}\right)\left(i d_{\delta} \otimes R^{\bar{\gamma} \beta} \otimes i d_{\alpha}\right) \tilde{s} \\
& \quad=\left[i d_{\gamma} \otimes\left(s_{\delta \alpha} R^{\bar{\alpha} \delta} s_{\delta \alpha}\right)^{-1} \otimes i d_{\beta}\right]\left[s_{\alpha \gamma} R^{\bar{\gamma} \bar{\alpha}} s_{\alpha \gamma} \otimes s_{\beta \delta} R^{\delta \beta} s_{\beta \delta}\right]\left[i d_{\alpha} \otimes s_{\beta \gamma} R^{\bar{\gamma} \beta} s_{\beta \gamma} \otimes i d_{\delta}\right] \\
& \quad=\overline{S^{\alpha, \beta ; \gamma, \delta}} .
\end{aligned}
$$

Thus (1.13)' is fulfilled and by the previous remark it ends the proof of Theorem.

We denote

$$
\begin{gathered}
F u n\left(S L_{q}\left(N, \mathbb{C} ; \varepsilon_{1}, \ldots, \varepsilon_{N}\right)\right)=\operatorname{Fun}\left(S U_{q}\left(N ; \varepsilon_{1}, \ldots, \varepsilon_{N}\right)\right)^{\mathbb{C R}}, \\
F u n\left(O_{q}\left(N, \mathbb{C} ; \varepsilon_{1}, \ldots, \varepsilon_{N}\right)\right)=\operatorname{Fun}\left(O_{q}\left(N ; \varepsilon_{1}, \ldots, \varepsilon_{N}\right)\right)^{\mathbb{C R}}, \\
F u n\left(S p_{q}\left(n, \mathbb{C} ; \varepsilon_{1}, \ldots, \varepsilon_{2 n}\right)\right)=\operatorname{Fun}\left(S p_{q}\left(n ; \varepsilon_{1}, \ldots, \varepsilon_{2 n}\right)\right)^{\mathbb{C R}}
\end{gathered}
$$

The description of these CQT *-Hopf algebras is given by Theorem 3.1, Corollary 3.2 and Theorem 3.3. In particular, combining Corollary 3.2.3 with (2.29), we get

\section{Proposition 3.6.}

1) $F u n\left(S L_{q}\left(N, \mathbb{C} ; \varepsilon_{1}, \ldots, \varepsilon_{N}\right)\right)$ is the universal *-algebra with unity generated by $w_{i j}, i, j=1, \ldots, N$, satisfying

$$
\begin{gathered}
w^{\oplus N} E(q)=E(q) w^{\oplus 0}, \quad E(q)^{*} w^{\oplus N}=w^{\oplus 0} E(q)^{*}, \quad R(q)(w \oplus w)=(w \oplus w) R(q), \\
R^{\prime}(q)(\bar{w} \oplus w)=(w \oplus \bar{w}) R^{\prime}(q),
\end{gathered}
$$

where

$$
R^{\prime}(q)_{i j, k l}=\varepsilon_{k} \varepsilon_{j} R\left(q^{-1}\right)_{i k, j l}, \quad i, j, k, l=1, \ldots, N,
$$

$E(q), R(q)$ are given by $(2.4)-(2.5)$ and $w=\left(w_{i j}\right)_{i, j=1}^{N}, q>0$ (for $N$ odd we admit also $q<0)$.

2) $\operatorname{Fun}\left(O_{q}\left(N, \mathbb{C} ; \varepsilon_{1}, \ldots, \varepsilon_{N}\right)\right)$ and $F u n\left(S p_{q}\left(n, \mathbb{C} ; \varepsilon_{1}, \ldots, \varepsilon_{2 n}\right)\right)$ are the universal *-algebras with unity generated by $w_{i j}, i, j=1, \ldots, N$, satisfying

$$
\begin{array}{cl}
(w \oplus w) C(q)=C(q) w^{\oplus 0}, & C(q)^{*}(w \oplus w)=w^{\oplus 0} C(q)^{*}, \\
R(q)(w \oplus w)=(w \oplus w) R(q), & R^{\prime}(q)(\bar{w} \oplus w)=(w \oplus \bar{w}) R^{\prime}(q),
\end{array}
$$

where

$$
R^{\prime}(q)_{i j, k l}=\varepsilon_{k} \varepsilon_{j} R\left(q^{-1}\right)_{i k, j l}, \quad i, j, k, l=1, \ldots, N,
$$

$C(q), R(q)$ are given by $(2.10)-(2.11)$ and $w=\left(w_{i j}\right)_{i, j=1}^{N}, q \in \mathbb{R} \backslash\{0\}\left(q>0\right.$ for $\left.B_{n}\right)$.

3) In both cases $w=\psi^{\otimes i d}(u), \tau^{\otimes i d}(w)=u$, where $u$ is given in Ex. 2.1 or Ex. 2.2. 
Remark 4. For $q=1, R_{i j, k l}^{\prime}=R_{i j, k l}=\delta_{i l} \delta_{j k}, i, j, k, l=1, \ldots, N$, thus dependence on $\varepsilon_{1}, \ldots, \varepsilon_{N}$ vanishes and we get relations, which are satisfied for the classical groups $S L(N, \mathbb{C}), O(N, \mathbb{C})$ (taken in a suitable form) and $\operatorname{Sp}(n, \mathbb{C})$.

Remark 5. An explicit form of (3.76) is given by

$$
\begin{gathered}
w_{k l}{ }^{*} w_{i j}=w_{i j} w_{k l}^{*}, \quad i \neq k, j \neq l, \\
q w_{k j}{ }^{*} w_{i j}=w_{i j} w_{k j}{ }^{*}+\left(1-q^{2}\right) \sum_{l<j} \varepsilon_{l} \varepsilon_{j} w_{i l} w_{k l}^{*}, \quad i \neq k, \\
w_{i k}{ }^{*} w_{i j}+\left(1-q^{2}\right) \sum_{l>i} \varepsilon_{i} \varepsilon_{l} w_{l k}{ }^{*} w_{l j}=q w_{i j} w_{i k}{ }^{*}, \quad j \neq k, \\
w_{i j}^{*} w_{i j}+\left(1-q^{2}\right) \sum_{l>i} \varepsilon_{i} \varepsilon_{l} w_{l j}{ }^{*} w_{l j}=w_{i j} w_{i j}{ }^{*}+\left(1-q^{2}\right) \sum_{k<j} \varepsilon_{k} \varepsilon_{j} w_{i k} w_{i k}{ }^{*},
\end{gathered}
$$

$i, j, k, l=1, \ldots, N$. Let $\varepsilon_{1}=\varepsilon_{2}=\cdots=\varepsilon_{N}=1$. Then after applying $\tau$, (3.77)(3.80) coincide with (4) of $[\mathrm{Br}]$.

Remark 6. Combining Thm. 3.1 with Remark 7 of Sec. 2, we get the representation theory of $\operatorname{Fun}\left(S L_{q}\left(N, \mathbb{C} ; \varepsilon_{1}, \ldots, \varepsilon_{N}\right)\right), q \in(0,1]$.

Remark 7. Consider $\mathbb{A}=F u n\left(S U_{q}(2 ; 1,1)\right)=F u n\left(S U_{q}(2)\right), q \in(0,1)$. There exists an invertible $Q \in \operatorname{Mor}(\bar{u}, u)$. Then (see (2.29)) $R^{\prime}(q)=\mathbb{R}^{\bar{u} u}=\left(i d \otimes Q^{-1}\right) R(Q \otimes i d)$ and (3.76) is equivalent to $R(\tilde{w} \oplus w)=(w \oplus \tilde{w}) R$ (where $\tilde{w}=Q \bar{w} Q^{-1}$ ), which coincides with (1.5) of [PW] $\left(q, \mathbb{R}, \tilde{w}\right.$ are, in notation of [PW], $\left.\mu, \mu^{1 / 2} \lambda, \bar{w}\right)$. Therefore $F u n\left(S L_{q}(2, \mathbb{C} ; 1,1)\right)$ can be identified with the *-algebra of $S L_{q}(2, \mathbb{C})$ given by relations (1.9)-(1.25) of [PW] (in the present paper we assumed $q>0$ in the case of $A_{1}$ ). The CQT $*-H o p f$ algebra structure on that object is given by $\mathrm{Thm} .3 .3$ (it would be interesting to compare it with the structure presented in Appendix of [Ta 2]). Let us consider the representation $T=\overline{w^{c}}(1) w$ of $F u n\left(S L_{q}(2, \mathbb{C} ; 1,1)\right)$. After some calculations one finds the corresponding $R$ matrix

$$
S^{T T}=(i d \otimes R \otimes i d)(R \otimes R)\left(i d \otimes R^{-1} \otimes i d\right) .
$$

The same matrix (up to a scalar multiple) was provided by eq. $\mathbb{I I} .38$ of [CSSW].

Remark 8. Some notions similar to those of this Section, but on the dual level, were investigated in [RS]. Using some element $R \in B \otimes A$, the authors define the twisted product Hopf algebra $A \otimes_{R} B$ of two Hopf algebras $A$ and $B$. They notice that $A \otimes_{R} A$ is (under some condition) quasitriangular, with universal $R$-matrix given by relation (2.26) of $[\mathbb{R S}]$, which is similar to (3.34) (cf. also Remark 7 and [Sch]). The paper [RS] doesn't use the conjugate Hopf algebras. 
Remark 9. S. L. Woronowicz noticed already in 1990 (the abstract of the lecture 'Non-compact quantum groups', ICM-1990) that 'In principle (at least on the $*$-Hopf algebra level) there exists the third one parameter deformation of the Lorentz group that can be obtained by applying the double group construction to quantum $S U(1,1)$ group.'

\section{A. Appendix}

Here we recall the basic notions and facts concerning (*-)Hopf algebras (cf. e.g. [Abe], [Swe], [W2], [Dr], [Ta 1], [So]).

Definition A.1. We say that $\mathbb{A}=(A, \phi, \kappa, e)$ is a Hopf algebra if $A$ is an algebra over $\mathbb{C}$ with unity $I, \phi: A \rightarrow A \otimes A$ and $e: A \rightarrow \mathbb{C}$ are unital homomorphisms, $\kappa: A \rightarrow A$ is a linear mapping and

$$
\begin{gathered}
(\phi \otimes i d) \phi=(i d \otimes \phi) \phi, \\
(e \otimes i d) \phi=(i d \otimes e) \phi=i d, \\
m(\kappa \otimes i d) \phi(x)=m(i d \otimes \kappa) \phi(x)=e(x) I,
\end{gathered}
$$

where $m: A \otimes A \rightarrow A$ denotes the multiplication map.

It is known that $e$ and $\kappa$ are unique for given $A, \phi$. Next, for any Hopf algebra, $\kappa$ is a unital antihomomorphism such that

$$
s_{A A}(\kappa \otimes \kappa) \phi=\phi \kappa, \quad e \kappa=e
$$

(cf. (0.2)). Moreover, if a linear mapping $\kappa_{1}: A \rightarrow A$ satisfies (A.3) with $m$ replaced by $m \circ s_{A A}$, then $\kappa_{1}=\kappa^{-1}$.

We say that $v=\left(v_{i j}\right)_{i, j=1}^{K} \in M_{K}(A)$ is a corepresentation of a Hopf algebra $A$ if

$$
\begin{gathered}
\phi v_{i j}=\sum_{k=1}^{K} v_{i k} \otimes v_{k j}, \\
e\left(v_{i j}\right)=\delta_{i j},
\end{gathered}
$$

$i, j=1,2, \ldots, K, K \in \mathbb{N}$. We write $\operatorname{dim} v=K$. Using (A.3) for $x=v_{i j}$, we get that $v$ is invertible (as a matrix) and $\left(v^{-1}\right)_{i j}=\kappa\left(v_{i j}\right), i, j=1, \ldots, \operatorname{dim} v$. Let

$$
\left(v^{c}\right)_{i j}=\kappa\left(v_{j i}\right), \quad i, j=1, \ldots, \operatorname{dim} v .
$$

Due to (A.4), $v^{c}$ is a corepresentation, called contragradient to $v$. One-dimensional corepresentation $1=(I)$ is called trivial.

We say that $S \in M_{L \times K}(\mathbb{C})$ intertwines corepresentations $v=\left(v_{i j}\right)_{i, j=1}^{K}$ and $w=\left(w_{m n}\right)_{m, n=1}^{L}$ if $S v=w S$. Then we write $S \in \operatorname{Mor}(v, w)$. If $\operatorname{Mor}(v, w) \subset$ $M_{L \times K}(\mathbb{C}) \cong \operatorname{Lin}\left(\mathbb{C}^{K}, \mathbb{C}^{L}\right)$ contains an injective (invertible, resp.) element then $v$ 
is called a subcorepresentation of (equivalent to, resp.) $w$. We write $v \simeq w$ if $v$ and $w$ are equivalent. Direct sums and tensor products (0.4) of corepresentations (treated as matrices) are corepresentations.

A corepresentation $v$ is called irreducible if $\operatorname{dim} v>0$ and $v$ has no subcorepresentations $w$ such that $0<\operatorname{dim} w<\operatorname{dim} v$. We say that a corepresentation is completely reducible (CR) if it is equivalent to a direct sum of irreducible ones.

Let $I$ be the set of all nonequivalent irreducible corepresentations of A. Then

$$
\left\{v_{i j}\right\}_{i, j=1, \ldots, \operatorname{dim} v, v \in I}
$$

are linearly independent (use the argument in the proof of Prop. 4.7 of [W2]). On the other hand, matrix elements of corepresentations linearly generate $A$. Therefore, if $A$ is $\mathrm{CR}$ (i.e., all corepresentations of $\mathbf{A}$ are $\mathrm{CR}$ ) then the elements (A.6) form a linear basis in $A$. Moreover, we have

Proposition A.2 (cf. Prop. 4.1 of [W5]). The elements (A.6) form a linear basis in $A \Leftrightarrow \mathbf{A}$ is $\mathbf{C R}$

Proof. ' $\Leftarrow$ ' has been already shown.

$\Rightarrow$ : Let $v \in \operatorname{Rep} \mathbf{A}$. Then the matrix elements of $v$ are linear combinations of the matrix elements of $u^{\alpha}, \alpha \in I_{0}$, where $I_{0}$ is a finite subset of $I$. Therefore the mapping $\varphi: \bigoplus_{\alpha \in I_{0}} M_{d_{\alpha}}(\mathbb{C}) \rightarrow M_{d}(\mathbb{C})\left(d_{\alpha}=\operatorname{dim} u^{\alpha}, d=\operatorname{dim} v\right)$ given by

$$
\varphi\left[\bigoplus_{\alpha \in I_{0}} \rho^{\otimes i d}\left(u^{\alpha}\right)\right]=\rho^{\otimes i d}(v), \quad \rho \in A^{\prime},
$$

is well defined. One easily checks that $\varphi$ is a unital homomorphism of algebras (cf. algebra structure of $A^{\prime}$ below). Hence $\varphi\left(\bigoplus_{\alpha \in I_{0}} x_{\alpha}\right)=Q\left(\bigoplus_{s \in S} x_{i(s)}\right) Q^{-1}$ for some finite set $S$, mapping $i: S \rightarrow I_{0}$ and bijective linear map $Q: \bigoplus_{s \in S} \mathbb{C}^{d_{i(s)}} \rightarrow$ $\mathbb{C}^{d}$. Comparing that with (A.7), we get $v=Q\left(\bigoplus_{s \in S} u^{i(s)}\right) Q^{-1}$.

Let $\operatorname{Rep} \mathbf{A}$ be the set of all corepresentations of $\mathbf{A}$. We say that a subset $L \subset \operatorname{Rep} \mathbf{A}$ is sufficient if the matrix elements of the corepresentations belonging to $L$ linearly generate $A$.

Let $L \subset \operatorname{Rep} \mathbf{A}$. We say that $v \in \bar{L}$ if $v \in \operatorname{Rep} \mathbb{A}$ and there exist a finite set $D$ and $l_{m} \in L, a_{m} \in \operatorname{Mor}\left(l_{m}, v\right), b_{m} \in \operatorname{Mor}\left(v, l_{m}\right)(m \in D)$ such that

$$
i d_{v}=\sum_{m \in D} a_{m} b_{m}
$$

(cf. [W3]). Clearly $\overline{\bar{L}}=\bar{L}$ and $\bar{L}$ contains all direct sums and equivalent corepresentations of corepresentations belonging to $L$. 
Proposition A.3. Let A be a CR Hopf algebra and LCRep A be sufficient. Then $\bar{L}=\operatorname{Rep} \mathbf{A}$.

Proof. Decomposing elements of $L$ into irreducible components we obtain a set $I^{\prime}$ of irreducible corepresentations such that $I^{\prime} \subset \bar{L}$ and matrix elements of all $w \in I^{\prime}$ linearly generate $A$. Thus $I^{\prime}=I, I \subset \bar{L}$. Let $v \in \operatorname{Rep} \mathbf{A}$. Then $v$ is equivalent to a direct sum of elements of $I$ and $v \in \bar{L}=\bar{L}$.

For a given Hopf algebra A we can define several Hopf algebras related to it.

1) Tensor power $\mathbf{A}_{n}=\left(A_{n}, \phi_{n}, \kappa_{n}, e_{n}\right)(n=1,2, \ldots)$ is constructed as follows: $A_{n}=A \otimes \cdots \otimes A \quad(n$ times $), \quad \kappa_{n}=\kappa \otimes \cdots \otimes \kappa \quad(n$ times $), \quad e_{n}=e \otimes \cdots \otimes e \quad(n$ times),

$$
\phi_{n}\left(x_{1} \otimes \cdots \otimes x_{n}\right)=\sum_{i_{1}, \ldots, i_{n}} a_{i_{1}} \otimes \cdots \otimes a_{i_{n}} \otimes b_{i_{1}} \otimes \cdots \otimes b_{i_{n}},
$$

where $x_{1}, \ldots, x_{n} \in A$ and $a_{i_{1}}, \ldots, a_{i_{n}}, b_{i_{1}}, \ldots, b_{i_{n}}$ are elements of $A$ such that $\phi\left(x_{k}\right)=\sum_{i_{k}} a_{i_{k}} \otimes b_{i_{k}}, k=1, \ldots, n$ (finite sums). It is straightforward that $\mathbb{A}_{n}$ is a Hopf algebra.

2) Let $\mathbf{A}=(A, \phi, \kappa, e)$ be a Hopf algebra with bijective $\kappa, A^{j}$ be the conjugate algebra of $A, j: A \rightarrow A^{j}$ the corresponding unital antilinear antiisomorphism (if $a \in A$ then we denote $a^{j}=j(a)$ ), $\phi^{j}=(j \otimes j) \phi j^{-1}, \kappa^{j}=j \kappa^{-1} j^{-1}, e^{j}=\backsim e j^{-1}$ $\left(\sim\right.$ is the complex conjugation in $\mathbb{C}$ ). Then $\mathbf{A}^{j}=\left(A^{j}, \phi^{j}, \kappa^{j}, e^{j}\right)$ is a Hopf algebra and is called the conjugate Hopf algebra of $\mathbf{A}([\mathrm{Ko}])$.

3) Let us notice that $A^{\prime}$ (the dual vector space of $A$ ) is in a natural way an algebra with unity $\left(1_{A^{\prime}}=e, x \cdot y=(x \otimes y) \phi, x, y \in A^{\prime}\right)$. Set $\Delta=m^{\prime}$, $S=\kappa^{\prime}, \varepsilon=\eta^{\prime}$ (where $\eta: \mathbb{C} \rightarrow A$ is a linear mapping such that $\left.\eta(1)=I\right), \sigma=s_{A A}{ }^{\prime}$. In general $\mathbf{A}^{\prime}=\left(A^{\prime}, \Delta, S, \varepsilon\right)$ is not a Hopf algebra $\left(\operatorname{Im} \Delta \subset(A \otimes A)^{\prime} \supsetneqq A^{\prime} \otimes A^{\prime}\right.$ for $\operatorname{dim} A=\infty)$. Nevertheless, $\mathbf{A}^{\prime}$ satisfies all conditions of Definition A.1 with $\otimes$ replaced by $\otimes$ (see $(0.1)$; instead of $m$ we use $M=\phi^{\prime}\left(M_{\mid A^{\prime} \otimes A^{\prime}}\right.$ is the multiplication in $\left.A^{\prime}\right) ; \Delta, \varepsilon$ are homomorphisms in the following sense:

$$
\Delta M=(M \hat{\otimes} M)(i d \hat{\otimes} \sigma \hat{\otimes} i d)(\Delta \hat{\otimes} \Delta), \quad \varepsilon \hat{\otimes} \varepsilon=\varepsilon M ;
$$

$\left.i d \hat{\otimes} \sigma \hat{\otimes} i d=\left(i d \otimes s_{A A} \otimes i d\right)^{\prime}\right)$.

In particular, we can consider $\mathbf{A}_{n}{ }^{\prime}, n=1,2, \ldots$ In that context we use the following notation. Let $L \in A_{2}{ }^{\prime}=(A \otimes A)^{\prime}$. Then $L_{12}, L_{13}, L_{23} \in A_{3}{ }^{\prime}$ are defined by $L_{12}(x \otimes y \otimes z)=L(x \otimes y) e(z), L_{13}(x \otimes y \otimes z)=L(x \otimes z) e(y), L_{23}(x \otimes$ $y \otimes z)=e(x) L(y \otimes z), x, y, z \in A$.

Definition A.4. We say that $(A, *, \phi, \kappa, e)$ is a $*$-Hopf algebra if $(A, \phi, \kappa, e)$ is a Hopf algebra, $(A, *)$ is a $*$-algebra and $\phi *=(* \otimes *) \phi$.

In that situation $e$ is a $*$-homomorphism ( $\tilde{e}=\sim \circ e \circ *$ also satisfies (A.2), hence $\tilde{e}=e)$ while $\kappa \circ * \circ \kappa \circ *=i d(\tilde{\kappa}=* \circ \kappa \circ *$ satisfies (A.3) with $m$ re- 
placed by $m \circ s_{A A}$ ). Moreover, conjugations of corepresentations (see (0.5)) are corepresentations.

If $\mathbf{A}$ is a $*$-Hopf algebra, then $\mathbb{A}_{n}$ is also $\left(\left(x_{1} \otimes \cdots \otimes x_{n}\right)^{*_{n}}=x_{n}^{*} \otimes \cdots \otimes x_{1}^{*}\right.$, $x_{1}, \ldots, x_{n} \in A$ ). Moreover, $\mathbf{A}^{\prime}$ is in this case a $*$-algebra and (in an 'extended' sense: $\left.\Delta *_{A^{\prime}}=\left(*_{A^{\prime}} \otimes *_{A^{\prime}}\right) \Delta\right) \quad *$-Hopf algebra (we have two choices: $*_{A^{\prime}}(x)=$ $\sim \circ x \circ \kappa \circ *$ or $\left.*_{A^{\prime}}(x)=\backsim \circ x \circ * \circ \kappa, x \in A^{\prime}\right)$.

Let $\mathrm{A}=\left(A, \phi_{A}, \kappa_{A}, e_{A}\right)$ and $\mathrm{B}=\left(B, \phi_{B}, \kappa_{B}, e_{B}\right)$ be Hopf algebras. A unital homomorphism of algebras $\psi: A \rightarrow B$ is called a homomorphism of Hopf algebras $\mathbf{A}$ and $\mathbf{B}$ if $\phi_{B} \psi=(\psi \otimes \psi) \phi_{A}, e_{B} \psi=e_{A}$. Then $\kappa_{B} \psi=\psi \kappa_{A}$ (it can be checked on matrix elements of corepresentations). Moreover, if $*_{A}$ and $*_{B}$ endow $\mathbf{A}$ and $\mathbf{B}$ with the structure of $*$-Hopf algebras and $*_{B} \psi=\psi *_{A}$, then $\psi$ is called a homomorphism of $*-$ Hopf algebras $\mathbf{A}$ and $\mathbf{B}$.

\section{Acknowledgements}

I am very indebted to Professor T. H. Koornwinder for distributing of his notes $[\mathrm{Ko}]$, to which the present paper owes a lot. I would like to express my gratitude to Professor S. L. Woronowicz for his interest and support during preparation of this paper. I am also grateful to Professor H. Araki for his kind hospitality in RIMS. My stay here is supported by Monbusho Fellowship. The author is also thankful to Professors E. Abe, M. Takeuchi, Dr A. W. Schnizer and Dr D. S. McAnally for interesting discussions.

\section{References}

[Abe] Abe, E., Hopf algebras, Cambridge University Press, 1980.

[Br] Bra̧giel, K., The twisted $S U(N)$ group. On the $C^{*}$-algebra $C\left(S_{\mu} U(N)\right)$, Lett. Math. Phys., 20 (1990), 251-257.

[CSSW] Carow-Watamura, U., Schlieker, M., Scholl, M. and Watamura, S., Tensor representation of the quantum group $S L_{q}(2, \mathbb{C})$ and quantum Minkowski space, Z. Phys. $C$ Particles and Fields, 48 (1990), 159-165.

[Dr] Drinfeld, V. G., Quantum groups, in Proceedings ICM-1986, Berkeley, (1987), 798820.

[Ha] Hayashi, T., Quantum groups and quantum determinants, Nagoya Univ. preprint.

[J] Jimbo, M., A $q$-difference analogue of $U(\mathrm{~g})$ and the Yang-Baxter equation, Lett. Math. Phys., 10 (1985), 63-69.

[Ko] Koornwinder, T. H., Comments on $\S 1$ of P. Podleś and S. L. Woronowicz "Quantum deformation of Lorentz group", unpublished notes, September 1990.

[LT] Larson, R. G. and Towber, J., Two dual classes of bialgebras related to the concepts of "quantum group" and "quantum Lie algebra", preprint.

[Lyu] Lyubashenko, V.V., Hopf algebras and vectorsymmetries, Uspekhi Mat. Nauk, 41(5) (1986), 185-186, in Russian.

[Ma] Majid, S., Quasitriangular Hopf algebras and Yang-Baxter equations, Int. Journal of Modern Phys. A, 5(1) (1990), 1-91.

[P] Podleś, P., in preparation. 
[Pu] Pusz, W., private information.

[PW] Podleś, P. and Woronowicz, S. L., Quantum deformation of Lorentz group, Commun. Math. Phys., 130 (1990), 381-431.

[Re] Reshetikhin, N. Yu., Quantized universal enveloping algebras, the Yang-Baxter equation and invariants of links. I, LOMI preprint E-4-87. Cf. also Wenzl, H., Quantum groups and subfactors of type B,C and D, Commun. Math. Phys., 133 (1990), 383-432; Cheng, Y., Ge, M.L. and Xue, K., Yang-Baxterization of braid group representations, Commun. Math. Phys., 136 (1991), 195-208.

[Ro] Rosso, M., Algèbres enveloppantes quantifiées, groupes quantiques compacts de matrices et calcul différentiel non commutatif, Duke Math. J., 61(1) (1990), 11-40.

[RS] Reshetikhin, N. Yu. and Semenov-Tian-Shansky, M. A., Quantum $R$-matrices and factorization problems, J. Geom. Phys., 5 (1988), 533-550.

[RTF] Reshetikhin, N. Yu., Takhtadzyan, L. A. and Faddeev, L. D., Quantization of Lie groups and Lie algebras, Leningrad Math. J., 1(1) (1990), 193-225. (Russian original: Algebra i analiz, 1 (1989)). Cf. also Takhtajan, L. A., Lectures on quantum groups, in Introduction to quantum group and integrable massive models of quantum fields theory. Nankai Lectures on Mathematical Physics 1989, World Scientific.

[Sch] Schirrmacher, A., Aspects of quantizing Lorentz symmetry, preprint MPI-PTh/91-77, October 1991.

[So] Soibelman, Ja. S., Algebra of functions on a compact quantum group and its representations, Algebra $i$ analiz, 2(1), 190-212, in Russian.

[Swe] Sweedler, M. E., Hopf algebras, W. A. Benjamin Inc., New York, 1969.

[Ta 1] Takeuchi, M., Topological coalgebras, Journal of algebra, 97(2) (1985), 505-539.

[Ta 2] - Finite dimensional representations of the quantum Lorentz group, preprint.

[W1] Woronowicz, S. L., Twisted $S U(2)$ group. An example of a non-commutative differential calculus, Publ. RIMS, Kyoto Univ., 23 (1987), 117-181.

[W2] - Compact matrix pseudogroups, Commun. Math. Phys., 111 (1987), 613-665.

[W3] - Tannaka-Krein duality for compact matrix pseudogroups. Twisted $S U(N)$ groups, Inv. Math., 93 (1988), 35-76.

[W4] —, private information.

[W5] - New quantum deformation of $S L(2, \mathbb{C})$. Hopf algebra level, preprint. 
